\title{
CORRELATIONS OF SUMS OF TWO SQUARES AND OTHER ARITHMETIC FUNCTIONS IN FUNCTION FIELDS
}

\author{
LIOR BARY-SOROKER AND ARNO FEHM
}

\begin{abstract}
We investigate a function field analogue of a recent conjecture on autocorrelations of sums of two squares by Freiberg, Kurlberg and Rosenzweig, which generalizes an older conjecture by Connors and Keating. In particular, we provide extensive numerical evidence and prove it in the large finite field limit. Our method can also handle correlations of other arithmetic functions and we give applications to (function field analogues of) the average of sums of two squares on shifted primes, and to autocorrelations of higher divisor functions twisted by a quadratic character.
\end{abstract}

\section{INTRODUCTION}

We study function field analogues of conjectures on autocorrelations of sums of two squares.

1.1. Correlations of arithmetic functions. A basic statistical property of an arithmetic function $\psi$ is its mean value; that is, the asymptotic as $x \rightarrow \infty$ of

$$
\langle\psi(n)\rangle_{n \leq x}=\frac{1}{x} \sum_{n \leq x} \psi(n) .
$$

More information is given by the cross-correlations of arithmetic functions $\psi_{1}, \ldots, \psi_{k}$, which are defined at $\left(h_{1}, \ldots, h_{k}\right) \in \mathbb{Z}^{k}$ as the asymptotic as $x \rightarrow \infty$ of

$$
\left\langle\prod_{i=1}^{k} \psi_{i}\left(n+h_{i}\right)\right\rangle_{n \leq x}=\frac{1}{x} \sum_{n \leq x} \psi_{1}\left(n+h_{1}\right) \cdots \psi_{k}\left(n+h_{k}\right) .
$$

In the special case when all the $\psi_{i}$ are equal, we use the term autocorrelations. Some of the most famous theorems and problems in number theory are about these statistical properties for certain specific arithmetic functions, like the Hardy-Littlewood prime tuple conjecture, a quantitative version of the twin prime conjecture, which can be expressed in terms of the autocorrelations of the von Mangoldt function $\Lambda$.

1.2. Autocorrelation of sums of two squares. An integer $n$ is a sum of two squares if there exist $x, y \in \mathbb{Z}$ such that $n=x^{2}+y^{2}$; i.e., it is a norm of the Gaussian integer $x+i y \in \mathbb{Z}[i]$. We let

$$
b(n)= \begin{cases}1, & \text { if there exist } x, y \in \mathbb{Z} \text { such that } n=x^{2}+y^{2}, \\ 0, & \text { otherwise. }\end{cases}
$$


The study of the statistics of $b(n)$ has a long history: Already Landau [Lan08] gives the mean value of $b$ as

$$
\langle b(n)\rangle_{n \leq x}=\frac{1}{x} \sum_{n \leq x} b(n) \sim K \cdot \frac{1}{\sqrt{\log x}}, \quad x \rightarrow \infty,
$$

where

$$
K=\frac{1}{\sqrt{2}} \prod_{p \equiv 3(\bmod 4)}\left(1-p^{-2}\right)^{-1 / 2} \approx 0.764
$$

is the Landau-Ramanujan constant. The distribution of sums of two squares was then studied intensively through the behavior of $b$ in short intervals in many works by various authors, including [Hoo74, Iwa76, Hoo94], see the introduction of [BBF17, §1.2] for a brief history.

As for autocorrelations of $b$, one has lower and upper bounds of the right order of magnitude for the pair autocorrelations:

$$
\frac{1}{\log x} \ll\langle b(n) b(n+h)\rangle_{n \leq x} \ll \frac{1}{\log x} .
$$

For $h=1$, the upper bound was proved by Rieger [Rie65] while the lower bound by Indlekofer and Schwarz in [IS72, Ind74, Sch72] (see Kelly [Kel78] and Bantle [Ban86] for short interval versions of the lower bound in (1.6)). Hooley [Hoo74] proved (1.6) for general $h \neq 0$. As Hooley [Hoo71] asserts, determining the asymptotics of $\langle b(n) b(n+h)\rangle_{n \leq x}$ brings "much the same difficulties" as computing $\langle\Lambda(n) \Lambda(n+h)\rangle_{n \leq x}$, which is a special case of the aforementioned Hardy-Littlewood conjecture.

For triple autocorrelation, as mentioned by Cochrane and Dressler [CD87], it is trivial that there are infinitely many triples $(n-1, n, n+1)$ with $b(n-1) b(n) b(n+1)=1$ (since one triple like this generates another one, namely $\left.\left(n^{2}-1, n^{2}, n^{2}+1\right)\right)$ and they give an upper bound of the expected order of magnitude

$$
\langle b(n-1) b(n) b(n+1)\rangle_{n \leq x} \ll \frac{1}{(\log x)^{3 / 2}} .
$$

Hooley [Hoo73] finds infinitely many $n$ with $b(n) b\left(n+h_{1}\right) b\left(n+h_{2}\right)=1$ for any $h_{1}, h_{2}$.

In the study of Connors and Keating [CK97] on the two-point correlations in the quantum spectrum of the square billiard, they give a conjectural pair autocorrelation of $b$ at $h \neq 0$,

$$
\langle b(n) b(n+h)\rangle_{n \leq x} \sim W_{h} \cdot \frac{1}{\log x}, \quad x \rightarrow \infty
$$

where $W_{h}$ is an explicit constant depending on $h$ (cf. [Iwa76] for a contradicting conjecture in the case $h=1$ ), and they provide numerical data in support of their conjecture. Assuming (1.7), Smilansky [Smi13] calculates the second moment of the distribution of $b$ in short intervals of length $\lambda \sqrt{\log x}$ and shows it is consistent with a Poisson distribution. Freiberg, Kurlberg, and Rosenzweig [FKR17] give heuristics for higher level autocorrelations and conjecture that for a tuple $h=\left(h_{1}, \ldots, h_{k}\right)$ of pairwise distinct integers,

$$
\left\langle b\left(n+h_{1}\right) \cdots b\left(n+h_{k}\right)\right\rangle_{n \leq x} \sim \mathfrak{S}_{h} \cdot\langle b(n)\rangle_{n \leq x}^{k}, \quad x \rightarrow \infty
$$


with

$$
\mathfrak{S}_{h}=\prod_{p} \frac{\delta_{h}(p)}{\delta_{0}(p)}
$$

where the product runs over all primes, and

$$
\delta_{h}(p)=\lim _{\nu \rightarrow \infty} \frac{\#\left\{n \in \mathbb{Z} / p^{\nu} \mathbb{Z}: \forall i \exists a_{i}, b_{i} \text { s.t. } n+h_{i} \equiv a_{i}^{2}+b_{i}^{2} \bmod p^{\nu}\right\}}{p^{\nu}} .
$$

It can happen that there is a local obstruction, leading to $\mathfrak{S}_{h}=0$, e.g. when the $h_{i}$ cover all residue classes modulo 4 , but [FKR17] show that $\mathfrak{S}_{h}>0$ if $k \leq 3$. We should also note that (1.7) is the special case $k=2$ of (1.8), i.e. $W_{h}=\mathfrak{S}_{(0, h)}$, see [FKR17, Discussion 1.1]. Assuming (1.8), [FKR17, Theorem 1.4] deduces that the distribution of the number of sums of two squares in short intervals of typical length is indeed Poisson. The goal of this paper is to provide evidence for (1.8) by studying this problem in the function field setting.

1.3. Correlations in the function field setting. In this setting, we replace the ring of integers by the ring of polynomials $\mathbb{F}_{q}[T]$ over a finite field $\mathbb{F}_{q}$ with $q$ elements. The positive integers up to $x$ are modeled by the subset $M_{n, q} \subseteq \mathbb{F}_{q}[T]$ of monic polynomials of degree $n$ and the prime polynomials are the monic irreducible polynomials. See e.g. [Rud14] for the classical analogue of the prime number theorem and a survey of some of the recent work on number theory in function fields. Our arithmetic functions are complex valued functions $\psi$ on the monic polynomials $M_{q}=\bigcup_{n=1}^{\infty} M_{n, q}$. In a general point of view, our goal is to understand the cross-correlations of arithmetic functions $\psi_{1}, \ldots, \psi_{k}$ on $M_{q}$ at $\left(h_{1}, \ldots, h_{k}\right) \in \mathbb{F}_{q}[T]^{k}$,

$$
\left\langle\prod_{i=1}^{k} \psi_{i}\left(f+h_{i}\right)\right\rangle_{f \in M_{n, q}}=\frac{1}{q^{n}} \sum_{f \in M_{n, q}} \psi_{1}\left(f+h_{1}\right) \cdots \psi_{k}\left(f+h_{k}\right)
$$

as the parameter $q^{n}=\# M_{n, q}$ is large (and $n>\operatorname{deg}\left(h_{i}\right)$ for all $i$ to avoid technical difficulties). This parameter can be large, in particular, either when $n$ is much larger than $q$, which we call the large degree limit, or when $q$ is much larger than $n$, which we call the large finite field limit.

Typically, in the large degree limit, one knows no more than what is known in number fields assuming the Generalized Riemann Hypothesis ${ }^{1}$ (which is, of course, a theorem in function fields). In the large finite field limit one can often go much further than what can be done in the number field setting or in the large degree limit. An extensive study by several authors [ABR15, BB15, Car15, CR14, Pol08] has led to a complete understanding of (1.9) in this limit for the family of arithmetic functions depending on cycle structure (see [ABR15, Theorem 1.4]).

\footnotetext{
${ }^{1}$ There are, however, several exceptions to this typical phenomenon; see for example, [Ha106, Poo03].
} 
1.4. Sums of two squares in the function field setting. There is a recent series of works on a certain function field analogue of sums of two squares, which we now recall briefly: For $f \in M_{q}$ we let

$$
b_{q}(f)= \begin{cases}1, & \text { if } f=A^{2}+T B^{2}, A, B \in \mathbb{F}_{q}[T], \\ 0, & \text { otherwise. }\end{cases}
$$

i.e. we consider norms from the ring $\mathbb{F}_{q}[\sqrt{-T}]$, which we take as the analogue of $\mathbb{Z}[i]$. With this definition, Smilansky, Wolf and the first named author [BSW16] give asymptotics for $\left\langle b_{q}(f)\right\rangle_{f \in M_{n, q}}$ in the limits $q \rightarrow \infty$ and $n \rightarrow \infty$, and Gorodetsky [Gor16] extends this to

$$
\left\langle b_{q}(f)\right\rangle_{f \in M_{n, q}} \sim K_{q} \cdot \frac{1}{4^{n}}\left(\begin{array}{c}
2 n \\
n
\end{array}\right), \quad q^{n} \rightarrow \infty
$$

where

$$
K_{q}=\left(1-q^{-1}\right)^{-\frac{1}{2}} \prod_{\chi_{q}(P)=-1}\left(1-|P|^{-2}\right)^{-\frac{1}{2}}=1+O\left(q^{-1}\right)
$$

is an explicit constant depending only on $q$ (see Section 2.1 for notation). Moreover, Bank and the two authors [BBF17] determine the mean value of $b$ in short intervals in the limit $q \rightarrow \infty$. In the spirit of these works, we can formulate a function field analogue of (1.8) for autocorrelations of $b_{q}$ :

Conjecture 1.1. Fix $N \geq 1$ and $k \geq 1$. Then for $q$ an odd prime power, $n \geq N$, and $h_{1}, \ldots, h_{k} \in \mathbb{F}_{q}[T]$ of degree less than $N$ and pairwise distinct,

$$
\begin{aligned}
\left\langle\prod_{i=1}^{k} b_{q}\left(f+h_{i}\right)\right\rangle_{f \in M_{n, q}} & \sim \mathfrak{S}_{q, h} \cdot\left\langle b_{q}(f)\right\rangle_{f \in M_{n, q}}^{k} \\
& \sim \mathfrak{S}_{q, h} \cdot K_{q}^{k} \cdot \frac{1}{4^{n k}}\left(\begin{array}{c}
2 n \\
n
\end{array}\right)^{k}
\end{aligned}
$$

uniformly as $q^{n} \rightarrow \infty$, where $K_{q}$ is defined as in (1.12) and

$$
\mathfrak{S}_{q, h}=\prod_{\substack{P \in \mathbb{F}_{q}[T] \\ \text { monic irred. }}} \frac{\delta_{q, h}(P)}{\delta_{q, 0}(P)^{k}}
$$

with

$$
\delta_{q, h}(P)=\lim _{\nu \rightarrow \infty} \frac{\#\left\{f \in \mathbb{F}_{q}[T] /\left(P^{\nu}\right): \forall i \exists A_{i}, B_{i} f+h_{i} \equiv A_{i}^{2}+T B_{i}^{2} \bmod P^{\nu}\right\}}{|P|^{\nu}} .
$$

The constant $\mathfrak{S}_{q, h}$ comes from the same heuristics that led to the constant $\mathfrak{S}_{h}$ of (1.8), see Section 2 for details, so Conjecture 1.1 is in perfect analogy with (1.8). We note that the product $\mathfrak{S}_{q, h}$ always converges and $\mathfrak{S}_{q, h}>0$ if and only if there are no local obstructions (Corollary 2.14). 
1.5. Results and method. As mentioned before, the main goal of this work is to give evidence for (1.8) by studying its function field analogue, Conjecture 1.1. We show that the local factors $\delta_{q, h}(P)$ can be computed in theory and we carry out this computation in special cases like $k=2$. In Section 2.4, we provide numerical evidence that supports Conjecture 1.1, which is more extensive than what can be done in number fields, as there is a fast algorithm to compute the analogue of $b$. We then prove Conjecture 1.1 in the large finite field limit, where the main term can be explicitly computed:

Theorem 1.2. Fix $n \geq 3$ and $k \geq 1$. Then for $q$ an odd prime power and $h_{1}, \ldots, h_{k} \in \mathbb{F}_{q}[T]$ of degree less than $n$ and pairwise distinct,

$$
\begin{aligned}
\left\langle\prod_{i=1}^{k} b_{q}\left(f+h_{i}\right)\right\rangle_{f \in M_{n, q}} & =\mathfrak{S}_{q, h} \cdot\left\langle b_{q}(f)\right\rangle_{f \in M_{n, q}}^{k}+O_{n, k}\left(q^{-1 / 2}\right) \\
& =\mathfrak{S}_{h} \cdot \frac{1}{4^{n k}}\left(\begin{array}{c}
2 n \\
n
\end{array}\right)^{k}+O_{n, k}\left(q^{-1 / 2}\right)
\end{aligned}
$$

where the implied constant depends only on $n$ and $k$, and

$$
\mathfrak{S}_{h}=2^{k-\#\left\{h_{1}(0), \ldots, h_{k}(0)\right\}} .
$$

We would like to highlight an interesting phenomenon: In the previously mentioned results on arithmetic functions that depend only on the cycle structure, it was shown that in the large finite field limit they become independent; hence the correlation dependence on $h$ disappears and can be seen only in the error term, cf. [KR16]. However, here, the dependence on the $h_{i}$ is non-trivial and agrees with the heuristics. The simple form of $\mathfrak{S}_{h}$ (as opposed to $\mathfrak{S}_{q, h}$ ) can be read as saying that in the large finite field limit only the correlation modulo the prime $T$ remains, as $f \equiv A^{2}+T B^{2} \bmod T$ for some $A, B \in \mathbb{F}_{q}[T]$ if and only if $f(0)$ is a square in $\mathbb{F}_{q}$.

In fact we prove Theorem 1.2 for correlations in short intervals instead of $M_{n, q}$ (see Theorem 4.2) and we also phrase Conjecture 1.1 in this generality (see Conjecture 2.2). We derive Theorem 1.2 from a general result on correlations of arithmetic functions that depend on what we call signed factorization type. This result reduces the large finite field limit of these correlations to combinatorial problems in certain finite groups; namely, fiber products of hyperoctahedral groups. In Section 3, we explain this general result (Theorem 3.11) and its proof, the main part of which consists of showing that the Galois group of a certain polynomial with a few variable coefficients is a fiber product of hyperoctahedral groups. Section 4 then contains the combinatorics for $b_{q}$, leading to Theorem 1.2, as well as of a few other arithmetic functions. In particular, we compute (function field analogues of) the average of $b$ on shifted primes (Theorem 4.7), and autocorrelations of higher divisor functions twisted by a quadratic character (Theorem 4.12); see the corresponding sections for more on the history and motivation of these questions. 


\section{Conjectural correlation of sums of two squares}

2.1. Preliminaries. With the convention $\operatorname{deg} 0=-\infty$, the norm on $\mathbb{F}_{q}[T]$ is given by $|f|=q^{\operatorname{deg} f}$. A short interval around a polynomial $f_{0}$ of degree $\operatorname{deg} f_{0}=n$ is defined analogously to the short intervals of integers $|n-x|<x^{\epsilon}$, cf. [KR14]:

$$
I_{q}\left(f_{0}, \epsilon\right):=\left\{f \in \mathbb{F}_{q}[T]:\left|f-f_{0}\right|<\left|f_{0}\right|^{\epsilon}\right\}=\left\{f_{0}+\sum_{i<\epsilon n} a_{i} T^{i}: a_{0}, \ldots, a_{\lfloor\epsilon n\rfloor} \in \mathbb{F}_{q}\right\} .
$$

So, roughly speaking, $f \in I_{q}\left(f_{0}, \epsilon\right)$ if and only if $1-\epsilon$ fraction of its higher coefficients coincide with those of $f_{0}$.

Every prime polynomial $P \in M_{q}$ defines a unique $P$-adic valuation $v_{P}: \mathbb{F}_{q}(T) \rightarrow \mathbb{Z} \cup\{\infty\}$ with $v_{P}(P)=1$. The condition that a prime number $p$ is inert in $\mathbb{Z}[i]$ corresponds to the condition that a prime polynomial $P \in \mathbb{F}_{q}[T]$ is irreducible in $\mathbb{F}_{q}[\sqrt{-T}]$; equivalently, that $P\left(-T^{2}\right)$ is irreducible in $\mathbb{F}_{q}[T]$. Hence, one of the reasons why $b_{q}$ is a suitable function field analogue of $b$ is that it has a multiplicative description very similar to Fermat's multiplicative description of $b$ :

Proposition 2.1. Let $f \in M_{q}$. Then $b_{q}(f)=1$ if and only if $v_{P}(f) \equiv 0 \bmod 2$ for every prime polynomial $P \in \mathbb{F}_{q}[T]$ with $P\left(-T^{2}\right) \in \mathbb{F}_{q}[T]$ irreducible.

Proof. See [BSW16, Thm. 2.5].

If we denote by

$$
\chi_{q}: \mathbb{F}_{q}[T] \rightarrow\{ \pm 1,0\}, \quad \chi_{q}(f)= \begin{cases}0, & \text { if } f(0)=0 \\ 1, & \text { if } f(0) \in \mathbb{F}_{q}^{\times 2}, \\ -1, & \text { otherwise. }\end{cases}
$$

the quadratic Dirichlet character modulo $T$, then the condition that $P\left(-T^{2}\right)$ is irreducible is equivalent to $\chi_{q}(P)=-1$, see [BSW16, $\left.\S 2\right]$.

2.2. Heuristics. The notation of this and the following subsection is taken from a preliminary version of [FKR17], and all proofs follow the corresponding proofs there. For

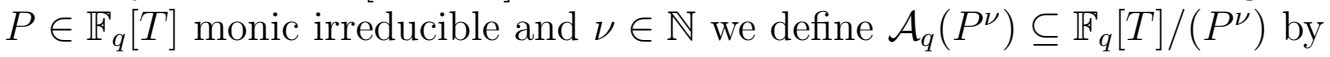

$$
\begin{aligned}
\mathcal{A}_{q}\left(P^{\nu}\right) & =\left\{f+\left(P^{\nu}\right): f \in M_{q}, b_{q}(f)=1\right\} \\
& =\left\{A^{2}+T B^{2}: A, B \in \mathbb{F}_{q}[T] /\left(P^{\nu}\right)\right\} .
\end{aligned}
$$

Moreover, for $h=\left(h_{1}, \ldots, h_{k}\right) \in \mathbb{F}_{q}[T]^{k}$ we let

$$
\mathcal{A}_{q, h}\left(P^{\nu}\right)=\left\{f \in \mathbb{F}_{q}[T] /\left(P^{\nu}\right): f+h_{1}, \ldots, f+h_{k} \in \mathcal{A}_{q}\left(P^{\nu}\right)\right\} .
$$

Note that if $f \in \mathcal{A}_{q, h}\left(P^{\nu}\right)$ then trivially $f \in \mathcal{A}_{q, h}\left(P^{\xi}\right)$ for all $\xi \leq \nu$, hence the sequence $|P|^{-\nu} \# \mathcal{A}_{q, h}\left(P^{\nu}\right)$ is monotone decreasing and therefore the limit

$$
\delta_{q, h}(P)=\lim _{\nu \rightarrow \infty}|P|^{-\nu} \# \mathcal{A}_{q, h}\left(P^{\nu}\right)
$$

exists. We will also show below (Corollary 2.14) that $\delta_{q, h}(P)=0$ if and only if $\mathcal{A}_{q, h}\left(P^{\nu}\right)=\emptyset$ for some $\nu>0$ in which case we say that there exists local obstruction at $P$. 
Our heuristic assumption is that the $b_{q}\left(f+h_{i}\right), i=1, \ldots, k$, behave like i.i.d. random variables as $f$ is randomly picked from a short interval $\left|f-f_{0}\right|<\left.\left|f_{0}\right|\right|^{\epsilon}$, up to a correction factor coming from the fact that they are not independent modulo polynomials $g$. By the Chinese Remainder Theorem, one may reduce to $g=P^{\nu}$ a prime power, for which the actual mean of $\prod_{i} b_{q}\left(f+h_{i}\right)$ is given by $\delta_{q, h}(P)$ while the random model would predict a mean of $\delta_{0}(P)^{k}$. This leads to

Conjecture 2.2. For every $k \geq 1, d \geq 1$ and $1 \geq \epsilon>0$ there exists $N \geq 1$ such that for $q$ an odd prime power, $f_{0} \in \mathbb{F}_{q}[T]$ monic of degree $n \geq N$ and $h_{1}, \ldots, h_{k} \in \mathbb{F}_{q}[T]$ of degree less than $d$ and pairwise distinct,

$$
\begin{aligned}
\left\langle\prod_{i=1}^{k} b_{q}\left(f+h_{i}\right)\right\rangle_{\left|f-f_{0}\right|<\left|f_{0}\right|^{\epsilon}} & \sim \mathfrak{S}_{q, h} \cdot\left\langle b_{q}(f)\right\rangle_{f \in M_{n, q}}^{k} \\
& \sim \mathfrak{S}_{q, h} \cdot K_{q}^{k} \cdot \frac{1}{4^{n k}}\left(\begin{array}{c}
2 n \\
n
\end{array}\right)^{k}
\end{aligned}
$$

uniformly as $q^{n} \rightarrow \infty$, where $\mathfrak{S}_{q, h}$ and $K_{q}$ are defined as in (1.13) resp. (1.12).

We note that if there exists a local obstruction, then clearly both sides in (2.2) equal 0 , so in this case the conjecture is uninteresting and correct.

2.3. The singular series. We now show that the $\delta_{q, h}(P)$ can be computed in theory and give estimates in general and concrete formulas in certain special cases. In particular, we show that the infinite product in (1.13) that defines $\mathfrak{S}_{q, h}$ indeed converges.

Lemma 2.3. Let $P \in \mathbb{F}_{q}[T]$ be monic irreducible and $f \in \mathbb{F}_{q}[T]$. Assume that $f \not \equiv 0$ $\bmod P^{\nu}$ and write $f \equiv P^{\alpha} g \bmod P^{\nu}$ with maximal $0 \leq \alpha<\nu$ and any suitable $g \in \mathbb{F}_{q}[T]$.

(1) If $\chi_{q}(P)=-1$, then $f \in \mathcal{A}_{q}\left(P^{\nu}\right)$ if and only if $\alpha$ is even.

(2) If $\chi_{q}(P)=0$, then $f \in \mathcal{A}_{q}\left(P^{\nu}\right)$ if and only if $\chi_{q}(g)=1$.

(3) If $\chi_{q}(P)=1$, then $f \in \mathcal{A}_{q}\left(P^{\nu}\right)$.

Proof. Note that $\mathcal{A}_{q}\left(P^{\nu}\right)$ is closed under multiplication and contains $P$ if $\chi_{q}(P) \in\{0,1\}$, and $P^{2}$ if $\chi_{q}(P)=-1$. If $\chi_{q}(P) \in\{ \pm 1\}$, then $g \in \mathcal{A}_{q}\left(P^{\nu}\right)$ : This follows for example from the prime polynomial theorem in arithmetic progressions [Ros02] which gives a prime polynomial $Q$ with $Q \equiv g \bmod P^{\nu}$ and $Q \equiv 1 \bmod T$, hence $b_{q}(Q)=1($ Proposition 2.1). This proves (3) and the 'if' part of (1). For the 'only if' part of $(1)$, note that if $b_{q}(f)=1$ and $f \equiv P^{\alpha} g \bmod P^{\nu}$, then $v_{P}(f)=\alpha$ is even (Proposition 2.1). For the 'if' part of (2) we can again apply the prime polynomial theorem to get a prime $Q$ with $Q \equiv g \bmod P^{\nu}$, which then satisfies $\chi_{q}(Q)=\chi_{q}(g)=1$, hence $b_{q}(Q)=1$. The 'only if' part of (2) is obvious, since the lowest nonzero coefficient of $A^{2}+T B^{2}$ is a square.

Lemma 2.4. Let $P \in \mathbb{F}_{q}[T]$ be monic irreducible.

(1) If $\chi_{q}(P)=-1$, then

$$
\# \mathcal{A}_{q}\left(P^{\nu}\right)=|P|^{\nu}\left(1-\frac{1}{|P|+1}\right)+\left\{\begin{array}{ll}
\frac{|P|}{|P|+1}, & \nu \text { odd } \\
\frac{1}{|P|+1}, & \nu \text { even }
\end{array} .\right.
$$


(2) If $\chi_{q}(P)=0$, then $\# \mathcal{A}_{q}\left(P^{\nu}\right)=\frac{|P|^{\nu}+1}{2}$.

(3) If $\chi_{q}(P)=1$, then $\# \mathcal{A}_{q}\left(P^{\nu}\right)=|P|^{\nu}$.

Proof. This follows by direct counting using Lemma 2.3. For example, if $\chi_{q}(P)=-1$ and $\nu$ is odd, then the nonzero elements of $\mathcal{A}_{q}\left(P^{\nu}\right)$ are represented by polynomials $\sum_{i=0}^{\nu-1} a_{i} P^{i}$ with $\operatorname{deg}\left(a_{i}\right)<\operatorname{deg}(P)$ and $\min \left\{i: a_{i} \neq 0\right\}$ even. Thus,

$$
\# \mathcal{A}_{q}\left(P^{\nu}\right)-1=\sum_{\alpha=0 \text { even }}^{\nu-1}(|P|-1)|P|^{\nu-\alpha-1}=|P|^{\nu-1}(|P|-1) \frac{1-|P|^{-\nu-1}}{1-|P|^{-2}},
$$

from which the claim follows.

Lemma 2.4 immediately gives $\delta_{q, h}(P)$ in the special case $h=0$ (which we identify with the 1-tuple $(0))$ or $\chi_{q}(P)=1$ :

Corollary 2.5. Let $P \in \mathbb{F}_{q}[T]$ monic irreducible. If $\chi_{q}(P)=1$, then $\delta_{q, h}(P)=1$.

Corollary 2.6. Let $P \in \mathbb{F}_{q}[T]$ monic irreducible. Then

$$
\delta_{q, 0}(P)= \begin{cases}1-\frac{1}{|P|+1}, & \chi_{q}(P)=-1 \\ \frac{1}{2}, & \chi_{q}(P)=0 \\ 1, & \chi_{q}(P)=1\end{cases}
$$

The computation of $\delta_{q, h}(P)$ in the rest of the cases is more technical. For $h=\left(h_{1}, \ldots, h_{k}\right)$ let

$$
\begin{aligned}
\Delta_{h} & =\prod_{i \neq j}\left(h_{i}-h_{j}\right), \\
\nu_{h}(P) & =\max _{i \neq j} v_{P}\left(h_{i}-h_{j}\right) .
\end{aligned}
$$

Lemma 2.7. Let $P \in \mathbb{F}_{q}[T]$ be monic irreducible. Let $h=\left(h_{1}, \ldots, h_{k}\right) \in \mathbb{F}_{q}[T]^{k}$ be a $k$-tuple of pairwise distinct polynomials. If $\chi_{q}(P)=-1$, then

$$
\left|\mathcal{A}_{q, h}\left(P^{\nu}\right)\right| \geq|P|^{\nu}\left(1-\frac{k}{|P|+1}\right)+k \cdot \begin{cases}\frac{|P|}{|P|+1}, & \nu \text { odd } \\ \frac{1}{|P|+1}, & \nu \text { even }\end{cases}
$$

with equality if $P \nmid \Delta_{h}$.

Proof. We use Lemma 2.4 and that $\mathcal{A}_{q, h}\left(P^{\nu}\right)=\bigcap_{i=1}^{k} \mathcal{A}_{q, h_{i}}\left(P^{\nu}\right)$ and $\# \mathcal{A}_{q, h_{i}}\left(P^{\nu}\right)=\# \mathcal{A}_{q}\left(P^{\nu}\right)$, to get that

$$
\# \mathcal{A}_{q, h}\left(P^{\nu}\right)=|P|^{\nu}-\#\left(\mathbb{F}_{q}[T] /\left(P^{\nu}\right) \backslash \mathcal{A}_{q, h}\left(P^{\nu}\right)\right) \geq|P|^{\nu}-k\left(|P|^{\nu}-\# \mathcal{A}_{q}\left(P^{\nu}\right)\right) .
$$

If $P \nmid \Delta_{h}$, then at most one of $f+h_{1}, \ldots, f+h_{k}$ is not in $\mathcal{A}_{q}\left(P^{\nu}\right)$ (by Lemma 2.3(1)), hence the complements of the $\mathcal{A}_{q, h_{i}}\left(P^{\nu}\right)$ are disjoint.

Proposition 2.8. If $\chi_{q}(P)=-1$, then

$$
\delta_{q, h}(P)=1-\frac{\eta}{|P|+1}
$$

where $1 \leq \eta \leq k$. Moreover, $\eta=1$ if $h=0$, and $\eta=k$ if $P \nmid \Delta_{h}$. 
Proof. Trivially, $\mathcal{A}_{q, h}\left(P^{\nu}\right) \subseteq \mathcal{A}_{q}\left(P^{\nu}\right)$, hence $\delta_{q, h}(P) \leq \delta_{q, 0}(P)$ is an upper bound, and $\delta_{q, 0}(P)=1-\frac{1}{|P|+1}$ by Corollary 2.6. The lower bound follows from Lemma 2.7.

We let

$$
\tilde{\mathfrak{S}}_{q, k}:=\prod_{\chi_{q}(P)=-1} \frac{1-\frac{k}{|P|+1}}{\delta_{q, 0}(P)^{k}}
$$

and observe that

$$
\mathfrak{S}_{q, h}=\frac{\delta_{q, h}(T)}{\delta_{q, 0}(T)^{k}} \cdot \prod_{P \mid \Delta_{h}, \chi_{q}(P)=-1} \frac{\delta_{q, h}(P)}{1-\frac{k}{|P|+1}} \cdot \tilde{\mathfrak{S}}_{q, k}
$$

by Proposition 2.8 and Corollary 2.5. So $\mathfrak{S}_{q, h}$ is convergent if and only if $\widetilde{\mathfrak{S}}_{q, k}$ is convergent.

Lemma 2.9. For every $k$, the product defining $\tilde{\mathfrak{S}}_{q, k}$ converges to a positive constant, and $\tilde{\mathfrak{S}}_{q, k}=1+O_{k}\left(q^{-1}\right)$.

Proof. For each prime polynomial $P$ put

$$
a_{P}=k \log \left(1-\frac{1}{|P|+1}\right)-\log \left(1-\frac{k}{|P|+1}\right) .
$$

By Bernoulli's inequality we have that $a_{P} \geq 0$ for all $P$, hence the series

$$
S_{q}:=\sum_{\chi_{q}(P)=-1} a_{P}
$$

in convergent if and only if it is bounded. Using the Taylor expansion $-\log (1-x)=$ $x+O\left(x^{2}\right)$, we have that

$$
a_{P} \ll|P|^{-2}=q^{-2 \operatorname{deg} P} .
$$

Since the number of $P$ of degree $d$ with $\chi_{q}(P)=-1$ is trivially no more than $q^{d}$, this gives

$$
S_{q} \ll \sum_{d=1}^{\infty} q^{d} q^{-2 d} \ll \frac{1}{q} .
$$

Thus the series is convergent and $S_{q}=O(1 / q)$. Now, as $\tilde{\mathfrak{S}}_{q, k}=\exp \left(-S_{q}\right)$, the product $\tilde{\mathfrak{S}}_{q, k}$ converges and $\tilde{\mathfrak{S}}_{q, k}=1+O(1 / q)$.

From Lemma 2.9 and (2.6) the following assertion immediately follows:

Corollary 2.10. Let $h=\left(h_{1}, \ldots, h_{k}\right) \in \mathbb{F}_{q}[T]^{k}$ be a $k$-tuple of pairwise distinct polynomials and let $\Delta_{h}$ as defined in (2.4). Then $\mathfrak{S}_{q, h}$ converges. Moreover $\mathfrak{S}_{q, h}=0$ if and only if there exists $P \mid T \Delta_{h}(T)$ with $\chi_{q}(P) \neq 1$ such that $\delta_{q, h}(P)=0$.

Next we give convenient formulas for $\mathfrak{S}_{q, h}$.

Lemma 2.11. Let $P \in \mathbb{F}_{q}[T]$ monic irreducible and $f \in \mathcal{A}_{q}\left(P^{\nu}\right)$.

(1) Assume $\chi_{q}(P)=-1$.

(a) If $f \not \equiv 0 \bmod P^{\nu}$ or $\nu$ is even, then $f \in \mathcal{A}_{q}\left(P^{\nu+1}\right)$. 
(b) If $f \equiv 0 \bmod P^{\nu}$ and $\nu$ is odd, then $f \in \mathcal{A}_{q}\left(P^{\nu+1}\right)$ if and only if $f \equiv 0$ $\bmod P^{\nu+1}$.

(2) Assume $\chi_{q}(P)=0$.

(a) If $f \not \equiv 0 \bmod P^{\nu}$, then $f \in \mathcal{A}_{q}\left(P^{\nu+1}\right)$.

(b) If $f \equiv 0 \bmod P^{\nu}$, then $f \equiv \alpha P^{\nu} \bmod P^{\nu+1}$ with $\alpha \in \mathbb{F}_{q}$, and $f \in \mathcal{A}_{q}\left(P^{\nu+1}\right)$ if and only if $\alpha=0$ or $\alpha \in \mathbb{F}_{q}^{\times 2}$.

Proof. This is immediate from Lemma 2.3.

We define

$$
\begin{aligned}
& \Omega_{q, h}\left(P^{\nu}\right)=\left\{f \in \mathcal{A}_{q, h}\left(P^{\nu}\right): \exists i f+h_{i} \equiv 0 \quad \bmod P^{\nu}\right\}, \\
& \Omega_{q, h}^{*}\left(P^{\nu}\right)=\left\{f \in \mathcal{A}_{q, h}\left(P^{\nu}\right): \forall i f+h_{i} \not \equiv 0 \quad \bmod P^{\nu}\right\} .
\end{aligned}
$$

Note that if $\nu>\nu_{h}(P)$, then $h_{1}+\left(P^{\nu}\right), \ldots, h_{k}+\left(P^{\nu}\right)$ are pairwise distinct, so $f+h_{i} \equiv 0$ $\bmod P^{\nu}$ for at most one $i$, and

$$
\# \Omega_{q, h}\left(P^{\nu}\right)=\#\left\{i: h_{j}-h_{i} \in \mathcal{A}_{q}\left(P^{\nu}\right) \text { for all } j\right\} .
$$

Lemma 2.12. Let $P \in \mathbb{F}_{q}[T]$ be monic irreducible and $f \in \mathcal{A}_{q}\left(P^{\nu}\right)$, let $h=\left(h_{1}, \ldots, h_{k}\right) \in$ $\mathbb{F}_{q}[T]^{k}$ be a $k$-tuple of pairwise distinct polynomials, and $\nu_{h}$ as defined in (2.5).

(1) Assume $\chi_{q}(P)=-1$.

(a) If $f \in \Omega_{q, h}^{*}\left(P^{\nu}\right)$, then $f \in \Omega_{q, h}^{*}\left(P^{\xi}\right)$ for all $\xi \geq \nu$.

(b) If $f \in \Omega_{q, h}\left(P^{\nu}\right)$ and $\nu>\nu_{h}(P)$, then $f+g P^{\nu} \in \Omega_{q, h}\left(P^{\nu+1}\right)$ for precisely one $g \in \mathbb{F}_{q}[T]$ with $\operatorname{deg}(g)<\operatorname{deg}(P)$. For all $g^{\prime} \neq g$ with $\operatorname{deg}\left(g^{\prime}\right)<\operatorname{deg}(P)$, $f+g^{\prime} P^{\nu} \in \Omega_{q, h}^{*}\left(P^{\nu+1}\right)$ if $\nu$ is even, and $f+g^{\prime} P^{\nu} \notin \mathcal{A}_{q, h}\left(P^{\nu+1}\right)$ if $\nu$ is odd.

(2) Assume $\chi_{q}(P)=0$.

(a) If $f \in \Omega_{q, h}^{*}\left(P^{\nu}\right)$, then $f \in \Omega_{q, h}^{*}\left(P^{\xi}\right)$ for all $\xi \geq \nu$.

(b) If $f \in \Omega_{q, h}\left(P^{\nu}\right)$ and $\nu>\nu_{h}(P)$, then $f+\alpha P^{\nu} \in \Omega_{q, h}\left(P^{\nu+1}\right)$ for one $\alpha \in \mathbb{F}_{q}$, $f+\alpha P^{\nu} \in \Omega_{q, h}^{*}\left(P^{\nu+1}\right)$ for $\frac{q-1}{2}$ many $\alpha \in \mathbb{F}_{q}$, and $f+\alpha P^{\nu} \notin \mathcal{A}_{q, h}\left(P^{\nu+1}\right)$ for $\frac{q-1}{2} \operatorname{many} \alpha \in \mathbb{F}_{q}$.

Proof. This follows by applying Lemma 2.11 to the $f+h_{i}$.

Proposition 2.13. Let $P \in \mathbb{F}_{q}[T]$ be monic irreducible, let $h=\left(h_{1}, \ldots, h_{k}\right) \in \mathbb{F}_{q}[T]^{k}$ be a $k$-tuple of pairwise distinct polynomials, and $\nu_{h}$ as in (2.5). Fix $\nu>\nu_{h}(P)$.

(1) If $\chi_{q}(P)=-1$, then

$$
\delta_{q, h}(P)= \begin{cases}|P|^{-\nu}\left(\# \Omega_{q, h}^{*}\left(P^{\nu}\right)+\frac{1}{|P|+1} \# \Omega_{q, h}\left(P^{\nu}\right)\right), & \nu \text { odd } \\ |P|^{-\nu}\left(\# \Omega_{q, h}^{*}\left(P^{\nu}\right)+\frac{|P|}{|P|+1} \# \Omega_{q, h}\left(P^{\nu}\right)\right), & \nu \text { even }\end{cases}
$$

(2) If $\chi_{q}(P)=0$, then

$$
\delta_{q, h}(P)=|P|^{-\nu}\left(\# \Omega_{q, h}^{*}\left(P^{\nu}\right)+\frac{1}{2} \# \Omega_{q, h}\left(P^{\nu}\right)\right) .
$$


Proof. (1): By Lemma 2.12(1), for each $\xi \geq \nu$, we have $\# \Omega_{q, h}\left(P^{\xi}\right)=\# \Omega_{q, h}\left(P^{\nu}\right)$ and

$$
\# \Omega_{q, h}^{*}\left(P^{\xi+1}\right)=|P| \cdot \# \Omega_{q, h}^{*}\left(P^{\xi}\right)+ \begin{cases}(|P|-1) \# \Omega_{q, h}\left(P^{\xi}\right) & \xi \text { even } \\ 0 & \xi \text { odd }\end{cases}
$$

so, as $\xi \rightarrow \infty,|P|^{-\xi} \# \Omega_{q, h}^{*}\left(P^{\xi}\right)$ tends to

$$
|P|^{-\nu} \# \Omega_{q, h}^{*}\left(P^{\nu}\right)+|P|^{-\nu} \# \Omega_{q, h}\left(P^{\nu}\right) \cdot(|P|-1) \cdot\left\{\begin{array}{ll}
\sum_{\mu=0}^{\infty}|P|^{-(2 \mu+1)} & \nu \text { even } \\
\sum_{\mu=1}^{\infty}|P|^{-2 \mu} & \nu \text { odd }
\end{array},\right.
$$

from which the claim follows, as $|P|^{-\xi}\left(\# \mathcal{A}_{q, h}\left(P^{\xi}\right)-\# \Omega_{q, h}^{*}\left(P^{\xi}\right)\right)=|P|^{-\xi} \# \Omega_{q, h}\left(P^{\nu}\right) \rightarrow 0$, since $\# \Omega_{q, h}\left(P^{\nu}\right) \leq k$.

(2): By Lemma 2.12(2), for each $\xi \geq \nu$, we have $\# \Omega_{q, h}\left(P^{\xi}\right)=\# \Omega_{q, h}\left(P^{\nu}\right)$ and

$$
\# \Omega_{q, h}^{*}\left(P^{\xi+1}\right)=|P| \cdot \# \Omega_{q, h}^{*}\left(P^{\xi}\right)+\frac{|P|-1}{2} \cdot \# \Omega_{q, h}\left(P^{\xi}\right),
$$

so, as $\xi \rightarrow \infty,|P|^{-\xi} \# \Omega_{q, h}^{*}\left(P^{\xi}\right)$ tends to

$$
|P|^{-\nu} \# \Omega_{q, h}^{*}\left(P^{\nu}\right)+|P|^{-\nu} \# \Omega_{q, h}\left(P^{\nu}\right) \cdot \frac{|P|-1}{2} \cdot \sum_{\mu=1}^{\infty}|P|^{-\mu}
$$

from which again the claim follows.

Note the striking similarity between Proposition 2.13(2) and the formula for $\delta_{\mathbf{k}}(2)$ in [FKR17]. As an immediate consequence of Corollary 2.6 and Proposition 2.13 we have

Corollary 2.14. There exists local obstruction at $P$ if and only if $\delta_{q, h}(P)=0$. In particular, $\mathfrak{S}_{q, h}=0$ if and only if there exists local obstruction at some prime $P$.

We now give explicit formulas for the case $k=2$; i.e. $h=\left(h_{1}, h_{2}\right)$. In this case, we may assume that $h$ is of the form $h=\left(0, h_{1}\right)$.

Proposition 2.15. For $h=\left(0, h_{1}\right)$ and $P \in \mathbb{F}_{q}[T]$ monic irreducible with $v_{P}\left(h_{1}\right)=\rho$ we have

$$
\delta_{q, h}(P)= \begin{cases}1-\frac{||^{\rho}+1}{|P|^{\rho}(|P|+1)}, & \chi_{q}(P)=-1 \\ \frac{1}{2}-\frac{1}{4|P|^{\rho}}-\frac{1}{4|P|^{\rho+1}}, & \chi_{q}(P)=0 \\ 1, & \chi_{q}(P)=1 .\end{cases}
$$

In particular, $\delta_{q, h}(P)>0$ for all $P$, so there exists no local obstruction in the case $k=2$.

Proof. Apply Proposition 2.13 with $\nu=\rho+1$. If $\chi_{q}(P)=-1$ and $\nu$ is odd, then $\# \Omega_{q, h}\left(P^{\nu}\right)=2$ and $\# \Omega_{q, h}^{*}\left(P^{\nu}\right)=\frac{|P|^{\nu}\left(1-|P|^{-\rho}\right)}{1+|P|^{-1}}+|P|-2$. If $\chi_{q}(P)=-1$ and $\nu$ is even, then $\# \Omega_{q, h}\left(P^{\nu}\right)=0$ and $\# \Omega_{q, h}^{*}\left(P^{\nu}\right)=\frac{|P|^{\nu}-1}{1+|P|^{-1}}$. If $\chi_{q}(P)=0$ and $h=T^{\rho} g$, then $\# \Omega_{q, h}\left(P^{\nu}\right)=1+\frac{1}{2}\left(\chi_{q}(g)+\chi_{q}(-g)\right)$ and $\# \Omega_{q, h}^{*}\left(P^{\nu}\right)=\frac{q^{\nu}}{2}-\frac{q}{2}+\frac{1}{4}\left(q-3-\chi_{q}(g)-\chi_{q}(-g)\right)$. 
Example 2.16. For $h=(0,1)$, we get

$$
\delta_{q, h}(P)= \begin{cases}1-\frac{2}{|P|+1}, & \chi_{q}(P)=-1 \\ \frac{1}{4}-\frac{1}{4|P|}, & \chi_{q}(P)=0 \\ 1, & \chi_{q}(P)=1\end{cases}
$$

For $h=(0, T)$, we get

$$
\delta_{q, h}(P)= \begin{cases}1-\frac{2}{|P|+1}, & \chi_{q}(P)=-1 \\ \frac{1}{2}-\frac{1}{4|P|}-\frac{1}{4|P|^{2}}, & \chi_{q}(P)=0 \\ 1, & \chi_{q}(P)=1\end{cases}
$$

In particular, we conclude that

$$
\frac{\mathfrak{S}_{q,(0, T)}}{\mathfrak{S}_{q,(0,1)}}=\frac{\delta_{q,(0, T)}(T)}{\delta_{q,(0,1)}(T)}=\frac{2 q^{2}-q-1}{q(q-1)}=2+\frac{1}{q},
$$

so Conjecture 2.2 predicts that

$$
\frac{\left\langle b_{q}(f) b_{q}(f+T)\right\rangle_{f \in M_{n, q}}}{\left\langle b_{q}(f) b_{q}(f+1)\right\rangle_{f \in M_{n, q}}} \sim 2+\frac{1}{q}, \quad q^{n} \rightarrow \infty .
$$

Remark 2.17. We note that, as opposed to the situation in integers, for small $q$ there can be a local obstruction in the case $k=3$, e.g. if $q=3$ and $h=(0,1,2)$, then $\delta_{q, h}(T)=0$.

Proposition 2.18. Fix $k \geq 1$ and $d \geq 1$. For $h_{1}, \ldots, h_{k} \in \mathbb{F}_{q}[T]$ pairwise distinct and of degree less than $d$,

$$
\mathfrak{S}_{q, h}=\mathfrak{S}_{h}+O_{k, d}\left(q^{-1 / 2}\right),
$$

where the implied constant depends only on $k$ and $d$, and $\mathfrak{S}_{q, h}$ and $\mathfrak{S}_{h}$ are defined as in (1.13) and (1.14).

Proof. Applying Lemma 2.9, Proposition 2.8, Corollary 2.6 to (2.6) and noting that $|P| \geq q$ gives

$$
\mathfrak{S}_{q, h}=\frac{\delta_{q, h}(T)}{\delta_{q, 0}(T)^{k}} \cdot \prod_{P \mid \Delta_{h}, \chi_{q}(P)=-1} \frac{\delta_{q, h}(P)}{1-\frac{k}{|P|+1}} \cdot \widetilde{\mathfrak{S}}_{q, k}=2^{k} \delta_{q, h}(T)+O\left(q^{-1}\right) .
$$

Let $S=\left\{h_{1}(0), \ldots, h_{k}(0)\right\}$ and $l=\# S$. Since on $\mathbb{F}_{q}^{\times}, \frac{\chi_{q}+1}{2}$ is the indicator function of $\mathcal{A}_{q}(T)$, by the definition of $\Omega_{q, h}^{*}$ in $(2.7)$ we can write

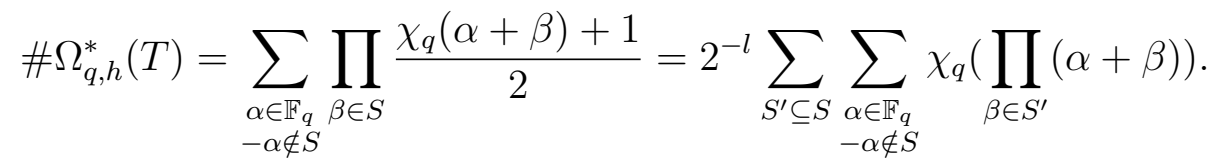

The Hasse-Weil theorem gives that $\sum_{\substack{\alpha \in \mathbb{F}_{q} \\-\alpha \notin S}} \chi_{q}\left(\prod_{\beta \in S^{\prime}}(\alpha+\beta)\right)=O\left(q^{-1 / 2}\right)$ when $S^{\prime} \neq \emptyset$. When $S^{\prime}=\emptyset$, one trivially has $\sum_{\substack{\alpha \in \mathbb{F}_{q} \\-\alpha \notin S}} \chi_{q}\left(\prod_{\beta \in S^{\prime}}(\alpha+\beta)\right)=q-l$. Thus, $\# \Omega_{q, h}^{*}(T)=$ 
$\frac{q-l}{2^{l}}+O\left(q^{1 / 2}\right)$ and

$$
q^{-\nu} \# \Omega_{q, h}^{*}\left(T^{\nu}\right)=q^{-\nu} \cdot\left(q^{\nu-1} \# \Omega_{q, h}^{*}(T)+O\left(q^{\nu-1}\right)\right)=\frac{1}{2^{l}}+O\left(q^{-1 / 2}\right) .
$$

By Proposition 2.13, $\delta_{q, h}(T)=2^{-l}+O\left(q^{-1 / 2}\right)$, as claimed.

We conclude this section by pointing out that $\delta_{q, h}(P)$ could be defined (and possibly computed) also in different ways, for example as the asymptotic density of

$$
\mathcal{A}_{q, h}\left(P^{\infty}\right)=\left\{f \in \mathbb{F}_{q}[T]: f+\left(P^{\nu}\right) \in \mathcal{A}_{q, h}\left(P^{\nu}\right) \text { for all } \nu\right\}
$$

like in [FKR17], or as the Haar measure of a suitable set in the completion of $\mathbb{F}_{q}[T]$ at $P$.

2.4. Numerics. In this section we compare Conjecture 2.2 with numerical computations. All our computation were done with the SageMath mathematics software system [Sage]. The algorithm to compute the function $b_{q}(f)$ is based on factoring $f$ into irreducibles and then applying Proposition 2.1. Here we exploit the fact that there is a fast factorization algorithm for $\mathbb{F}_{q}[T]$ implemented, as opposed to the situation in integers.

In the computations of the singular series, we need to compute $\delta_{q, h}(P)$, for which we apply Proposition 2.8 and Proposition 2.13, among other results.

We enumerate the polynomials in $\mathbb{F}_{q}[T]$ in the following order: $0,1, \ldots, T, T+1, \ldots$, and we will use this enumeration also in what follows.

2.4.1. Varying $h$. Conjecture 1.1 predicts that for each $h \in \mathbb{F}_{q}[T]$,

$$
\frac{\left\langle b_{q}(f) b_{q}(f+h)\right\rangle_{f \in M_{n, q}}}{\left\langle b_{q}(f) b_{q}(f+1)\right\rangle_{f \in M_{n, q}}} \sim \frac{\mathfrak{S}_{q,(0, h)}}{\mathfrak{S}_{q,(0,1)}}, \quad n \rightarrow \infty .
$$

In Figures 1 and 2, we compare this conjecture with numerics for $n=100, q=3$ and the 729 different $h$ of degree $<6$, enumerated as $h_{1}=0, h_{2}=1, \ldots, h_{729}=2 T^{5}+\cdots+2$ as indicated above. We have sampled $N=5 n^{2}=50000$ monic $f \in \mathbb{F}_{q}[T]$ of degree $n$ chosen at random and compute

$$
N_{1}\left(h_{i}\right)=\frac{\sum b_{q}(f) b_{q}\left(f+h_{i}\right)}{\sum b_{q}(f) b_{q}(f+1)} \quad \text { and } \quad H_{1}\left(h_{i}\right)=\frac{\mathfrak{S}_{q,\left(0, h_{i}\right)}}{\mathfrak{S}_{q,(0,1)}}
$$

where the sum in $N_{1}$ is taken over the sampled $f^{\prime}$ 's and $H_{1}\left(h_{i}\right)$ is computed precisely. Figure 2 is analogous to [CK97, Figure 2].

2.4.2. Varying $n$. For $h=\left(h_{1}, \ldots, h_{k}\right) \in \mathbb{F}_{q}[T]^{k}$ a $k$-tuple of pairwise distinct polynomials, Conjecture 1.1 predicts that

$$
\frac{\left\langle\prod_{i} b_{q}\left(f+h_{i}\right)\right\rangle_{f \in M_{n, q}}}{\left\langle b_{q}(f)\right\rangle_{f \in M_{n, q}}^{k}} \rightarrow \mathfrak{S}_{q, h}, \quad n \rightarrow \infty .
$$

We compare this conjecture with numerics for $q=5,3 \leq n<100$ and $h \in\{(0,1),(0, T)\}$ in Figure 3, and $h=(0,1, T)$ in Figure 4 . For each $n$ we have sampled $N=q n^{5 / 2}$ monic 

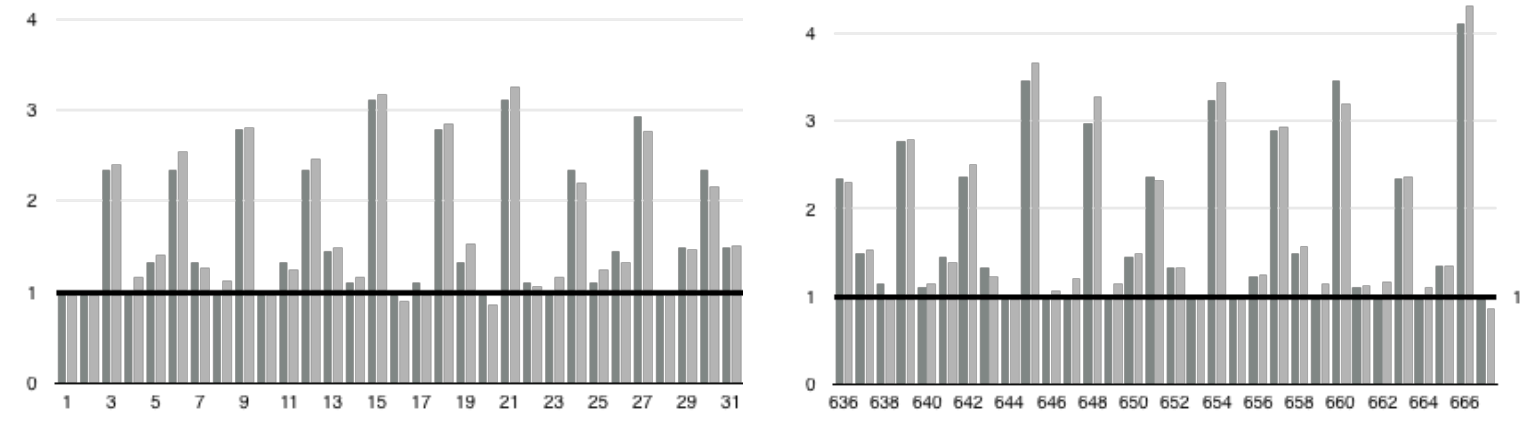

Figure 1. The plots show $N_{1}\left(h_{i}\right)$ (in light grey) and $H_{1}\left(h_{i}\right)$ (in dark grey) as functions of $i$ for the first 31 indices (in the left plot) and for a random starting point (in the right plot)

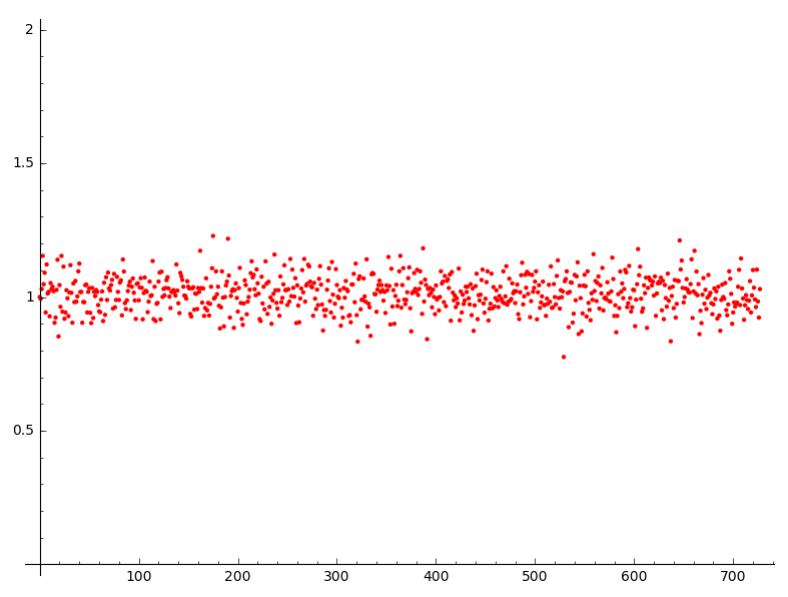

FiguRE 2. $\quad N_{1}\left(h_{i}\right) / H_{1}\left(h_{i}\right)$ as a function of $1 \leq i \leq 729$

$f \in \mathbb{F}_{q}[T]$ of degree $n$ chosen at random, and for each $h=\left(h_{1}, \ldots, h_{k}\right)$ as above we computed

$$
N_{2, h}(n)=\frac{N^{k-1} \sum \prod_{i=1}^{k} b_{q}\left(f+h_{i}\right)}{\left(\sum b_{q}(f)\right)^{k}}
$$

where the sums are taken over the sampled $f^{\prime}$ 's.

2.4.3. Varying q. Conjecture 1.1 (cf. (2.9)) suggests that

$$
\frac{\left\langle b_{q}(f) b_{q}(f+T)\right\rangle_{f \in M_{n, q}}}{\left\langle b_{q}(f) b_{q}(f+1)\right\rangle_{f \in M_{n, q}}}=2+\frac{1}{q}(1+o(1)), \quad q \rightarrow \infty .
$$

In Figure 5 we compare this conjecture with numerics. For each prime $2<q<30$ we evaluate the left hand side of $(2.12)$ by sampling random monic $f \in \mathbb{F}_{q}[T]$ of degrees 3 

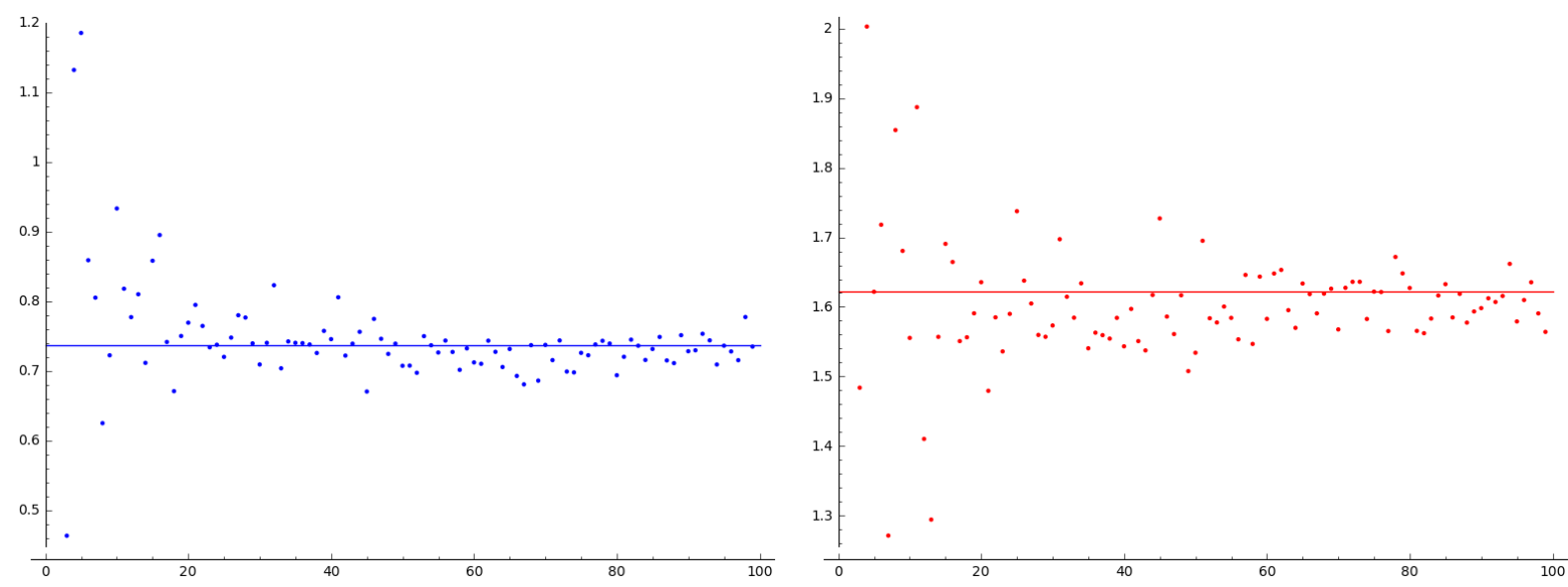

Figure 3 . The plots of $N_{2,(0,1)}(n)$ (on the left) and $N_{2,(0, T)}$ (on the right) as functions of $n$. The lines are approximated values of the respective singular series $\mathfrak{S}_{5,(0,1)}$ and $\mathfrak{S}_{5,(0, T)}$.

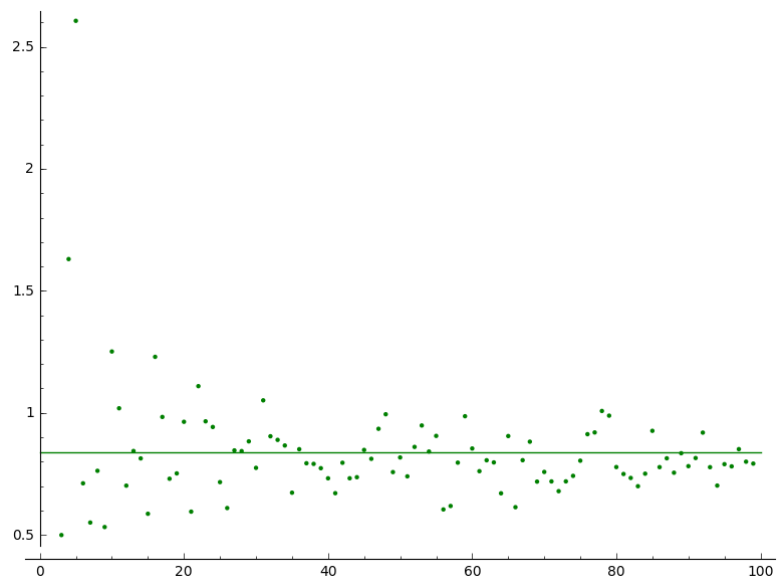

Figure $4 . N_{2,(0,1, T)}(n)$ as a function of $n$. The line is an approximated value of the respective singular series $\mathfrak{S}_{5,(0,1, T)}$.

up to 49 (and for $q=29$ up to 69 ), and this we denote by $N_{3}(q)$. We compare it to $H_{3}(q)=2+1 / q$.

2.4.4. Short intervals. We finally check Conjecture 2.2 about autocorrelations at $h=(0,1)$ in short intervals. Here we take $q=3$ and compute

$$
N_{4, f_{0}}(\epsilon)=\left\langle b_{q}(f) b_{q}(f+1)\right\rangle_{\left|f-f_{0}\right|<\left|f_{0}\right|^{\epsilon}}
$$

deterministically for fixed $f_{0}$ of degree $n \leq 150$ and various $\epsilon \leq 0.1$. Note that $N_{4, f_{0}}(\epsilon)$ is piecewise constant, namely constant on each interval $\frac{i}{n} \leq \epsilon<\frac{i+1}{n}$, and we chose one epsilon in each of these intervals, namely $\epsilon=\frac{i}{n}$. In Figure 6 we take two $f_{0}$ (namely $f_{0}=T^{150}$ 


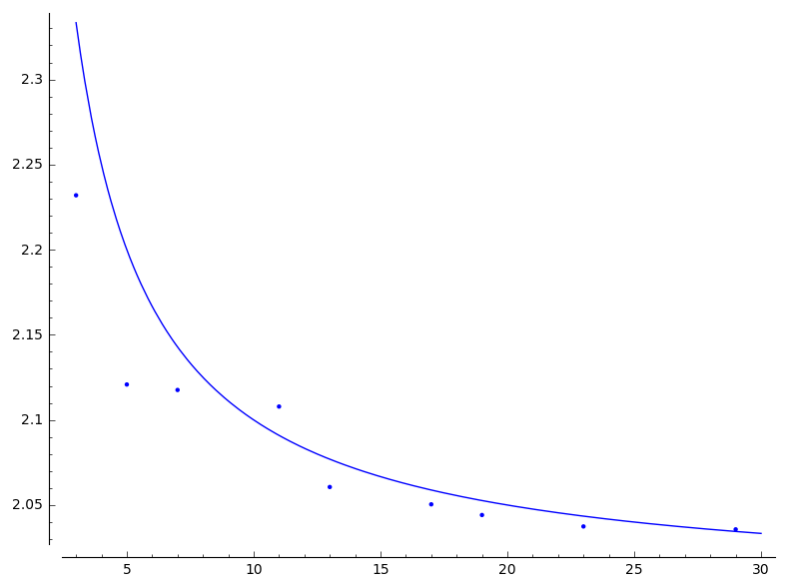

Figure 5. The curve is $H_{3}(q)=2+\frac{1}{q}$ as a function of $q$ and the points are $\left(q, N_{3}(q)\right)$ for primes $2<q<30$.
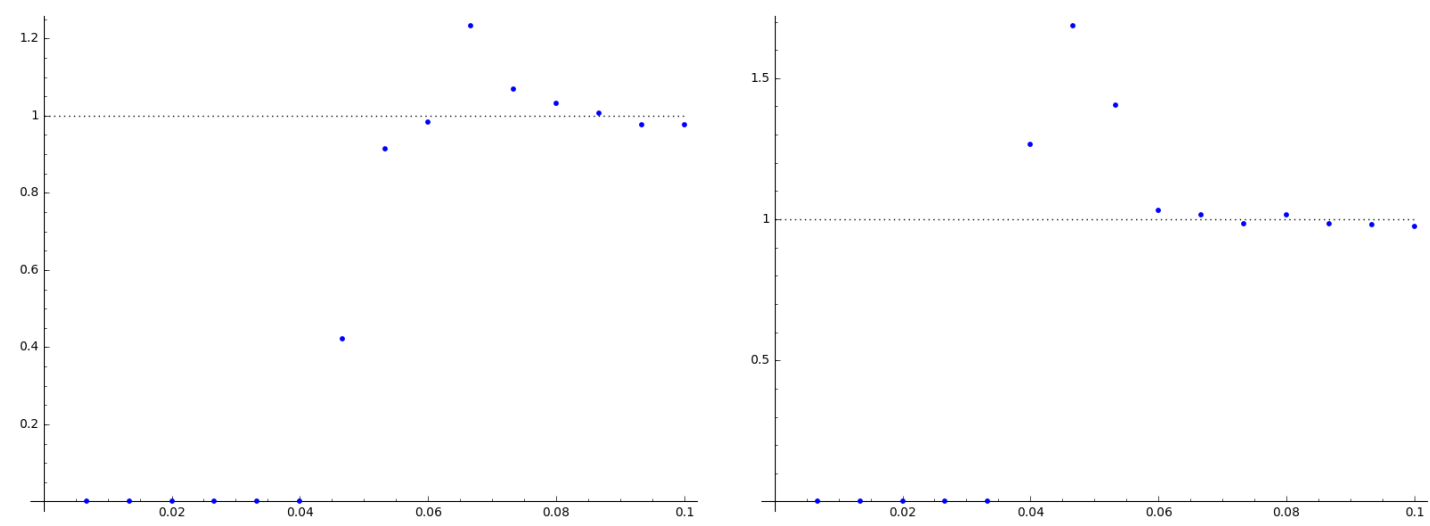

Figure 6. Here we plot $N_{4, f_{0}}(\epsilon) / H_{4, \operatorname{deg}\left(f_{0}\right)}$ as a function of $\epsilon$, for $f_{0}(T)=$ $T^{150}$ (in the left diagram) and a $f_{0}$ of degree 150 that is randomly chosen (in the right diagram).

and a $f_{0}$ of the same degree that is randomly chosen $^{2}$ ), and we vary $\epsilon$. In Figure 7 we take

\footnotetext{
${ }^{2} f_{0}=T^{150}+2 T^{149}+T^{146}+T^{144}+2 T^{143}+T^{140}+T^{138}+T^{137}+T^{136}+2 T^{135}+2 T^{133}+T^{132}+T^{130}+$ $2 T^{128}+2 T^{127}+T^{125}+2 T^{124}+2 T^{122}+2 T^{118}+T^{117}+T^{116}+2 T^{115}+T^{113}+T^{112}+2 T^{111}+2 T^{110}+$ $T^{109}+T^{106}+2 T^{103}+2 T^{102}+2 T^{100}+T^{98}+T^{97}+2 T^{96}+T^{95}+2 T^{94}+2 T^{91}+2 T^{90}+T^{88}+2 T^{87}+2 T^{86}+$ $2 T^{85}+T^{84}+2 T^{82}+T^{81}+T^{80}+2 T^{76}+T^{75}+T^{74}+T^{73}+2 T^{72}+2 T^{70}+T^{69}+2 T^{67}+T^{66}+2 T^{63}+T^{61}+$ $T^{59}+2 T^{58}+T^{57}+2 T^{56}+T^{52}+T^{51}+2 T^{50}+2 T^{49}+T^{48}+2 T^{47}+T^{46}+2 T^{44}+2 T^{43}+2 T^{40}+2 T^{39}+$ $T^{35}+T^{34}+2 T^{33}+T^{32}+2 T^{30}+2 T^{29}+2 T^{27}+2 T^{26}+T^{25}+T^{24}+T^{22}+T^{20}+T^{18}+2 T^{16}+2 T^{15}+$ $2 T^{14}+2 T^{13}+2 T^{12}+2 T^{11}+T^{10}+2 T^{9}+T^{8}+2 T^{7}+2 T^{6}+2 T^{3}+2 T^{2}+2 T+2$
} 

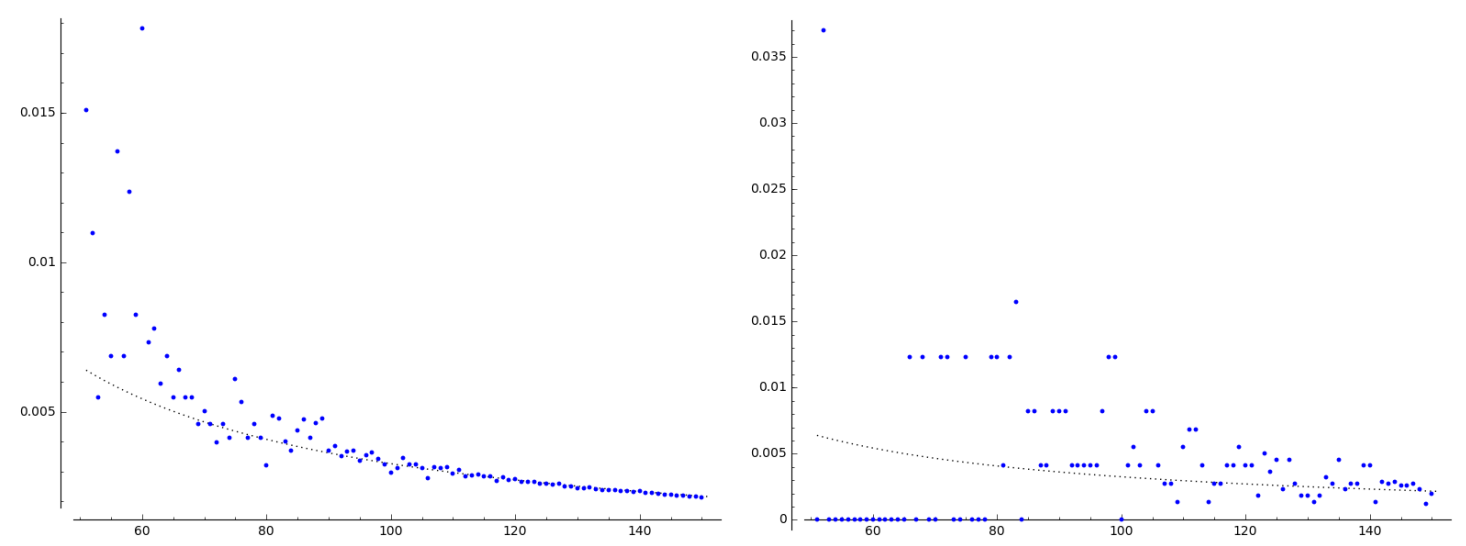

Figure 7. Here we plot $N_{4, T^{n}}(\epsilon)$ as a function of $n$, for $\epsilon=0.1$ (in the left diagram) and $\epsilon=0.05$ (in the right diagram), and compare it to $H_{4, n} \approx$ $0.3257 / n$.

$\epsilon=0.1$ and $\epsilon=0.05$, and we vary $n$. In each case, we compare $N_{4, f_{0}}(\epsilon)$ to the value

$$
H_{4, n}=\mathfrak{S}_{3,(0,1)} \cdot\left(K_{3} \cdot \frac{1}{4^{n}}\left(\begin{array}{c}
2 n \\
n
\end{array}\right)\right)^{2} \approx \frac{\mathfrak{S}_{3,(0,1)} K_{3}^{2}}{\pi n}
$$

predicted by Conjecture 2.2, where we use approximate values $\mathfrak{S}_{3,(0,1)} \approx 0.5736$ and $K_{3} \approx$ 1.3357. The observed quantization in the right diagram of Figure 7 is explained by the fact that here for small $n$ the short interval contains very few polynomials - for example for $60 \leq n<80$ just enough to contain either 0 or 1 element $f$ with $b_{q}(f) b_{q}(f+1)=1$.

\section{Arithmetic FunCtions of Signed faCtorization type}

In this section we define arithmetic functions depending on signed factorization type (see Section 3.1) and prove a general result (Theorem 3.11) for correlations of such functions in short intervals. This result explains the autocorrelations through distributions on finite groups. More precisely, it allows to compute the large finite field limit of an expression of the form

$$
\left\langle\prod_{i=1}^{k} \psi_{i}\left(f+h_{i}\right)\right\rangle_{\left|f-f_{0}\right|<\left|f_{0}\right|^{\epsilon}}
$$

as an average over corresponding functions on a certain finite group depending only on the combinatorics of $h_{1}(0), \ldots, h_{k}(0)$. In Section 3.1 we introduce arithmetic functions depending on signed factorization type, in Section 3.2 we explain the corresponding functions on finite groups and prove a general statement using a Chebotarev theorem. In Sections 3.3 and 3.4 we then study the finite groups that are relevant for correlations in short intervals, namely fiber products of hyperoctahedral groups, and in Section 3.5 we state and prove the general theorem. 
3.1. Signed factorization type. A signed factorization type is a function

$$
\lambda: \mathbb{N} \times \mathbb{N} \times\{ \pm 1,0\} \rightarrow \mathbb{Z}_{\geq 0}
$$

with finite support, and we denote by $\Lambda$ the set of all signed factorization types. For $\lambda \in \Lambda$ we let

$$
\operatorname{deg}(\lambda)=\sum_{d \in \mathbb{N}} \sum_{e \in \mathbb{N}} \sum_{s \in\{ \pm 1,0\}} \lambda(d, e, s) d e
$$

and

$$
\chi(\lambda)= \begin{cases}(-1)^{\sum_{d, e \in \mathbb{N}} \lambda(d, e,-1) e}, & \text { if } \lambda(d, e, 0)=0 \text { for all } d, e \\ 0, & \text { otherwise. }\end{cases}
$$

To each $f \in M_{q}$ with prime factorization $f=P_{1}^{e_{1}} \cdots P_{r}^{e_{r}}$ we assign a signed factorization type by setting

$$
\lambda_{f}(d, e, s):=\#\left\{i: \operatorname{deg}\left(P_{i}\right)=d, e_{i}=e, \chi_{q}\left(P_{i}\right)=s\right\}
$$

Note that

$$
\begin{array}{ll}
\operatorname{deg}\left(\lambda_{f}\right) & =\operatorname{deg}(f), \\
\chi\left(\lambda_{f}\right) & =\chi_{q}(f), \\
f \text { is square-free } & \Leftrightarrow \sum_{d, s} \sum_{e>1} \lambda_{f}(d, e, s)=0, \\
f(0) \neq 0 & \Leftrightarrow \sum_{d, e} \lambda_{f}(d, e, 0)=0 .
\end{array}
$$

We denote by $\Lambda^{*}$ the space of functions $\psi: \Lambda \rightarrow \mathbb{C}$. Each function $\psi \in \Lambda^{*}$ induces a family of arithmetic functions $\psi_{q}$ (for $q$ an odd prime power) on $M_{q}$ given by

$$
\psi_{q}: M_{q} \rightarrow \mathbb{C}, \quad f \mapsto \psi\left(\lambda_{f}\right) .
$$

Many arithmetic functions are of this form, in particular:

(1) The restriction of the quadratic character $\chi_{q}$ (see (2.1)) to $M_{q}$, which is induced from $\chi$ defined in (3.1).

(2) The indicator function of prime polynomials, which is induced from

$$
1_{\mathbb{P}}(\lambda)=\left\{\begin{array}{ll}
1, & \text { if } \sum_{d, s} \lambda(d, 1, s)=\sum_{d, e, s} \lambda(d, e, s)=1 \\
0, & \text { otherwise }
\end{array},\right.
$$

and the closely related function field analogue of the von Mangoldt function, see e.g. [KR14], which is induced from

$$
\Lambda(\lambda)= \begin{cases}d_{0}, & \text { if } \sum_{e, s} \lambda\left(d_{0}, e, s\right)=\sum_{d, e, s} \lambda(d, e, s)=1, \\ 0, & \text { otherwise }\end{cases}
$$

(3) The function $b_{q}$ defined in (1.10), which by Proposition 2.1 is induced from

$$
b(\lambda)= \begin{cases}1, & \text { if } \lambda(d, 2 e+1,-1)=0 \text { for all } d, e, \\ 0, & \text { otherwise }\end{cases}
$$

(4) The function field analogue of the Möbius function $\mu$, see e.g. [CR14]. 
(5) The function field analogue of the function $r$ counting the number of representations as sums of two squares, see Section 4.1.

(6) The function field analogue of the $r$-divisor function $d_{r}$, see Section 4.3.

3.2. Arithmetic functions on hyperoctahedral groups. The hyperoctahedral group (aka, signed permutation group) of degree $n$ is the permutational wreath product

$$
\mathrm{H}_{n}=\mathbb{F}_{2} \prec S_{n}=V \rtimes S_{n}
$$

with $V=\left(\mathbb{F}_{2}\right)^{n}$. The $S_{n}$-invariant subspaces of $V$ are

$$
\begin{array}{ll}
V_{n}=V, & V_{n-1}=\left\{x \in V: \sum_{i=1}^{n} x_{i}=0\right\}, \\
V_{1}=\{(0, \ldots, 0),(1, \ldots, 1)\}, & V_{0}=\{(0, \ldots, 0)\},
\end{array}
$$

cf. [BBF17, Lemma 4.2]. In particular, $V_{n-1} \rtimes S_{n}, V_{n} \rtimes A_{n}$ and $V_{n-1} \rtimes A_{n}$ are normal subgroups of $\mathrm{H}_{n}$. The total sign homomorphism

$$
\chi_{n}: \mathrm{H}_{n} \rightarrow\{ \pm 1\}
$$

is given by $\chi_{n}(x \tau)=(-1)^{\sum_{i} x_{i}}$, where $x=\left(x_{1}, \ldots, x_{n}\right) \in V$ and $\tau \in S_{n}$. It is obvious that $\chi_{n}$ is surjective and ker $\chi_{n}=V_{n-1} \rtimes S_{n}$.

To each $\sigma=x \tau \in \mathrm{H}_{n}$, with $x \in V$ and $\tau \in S_{n}$, we assign a signed factorization type by setting

$$
\lambda_{\sigma}(d, e, s)= \begin{cases}\#\left\{\Omega: \Omega \text { is an orbit of } \tau, \# \Omega=d,(-1)^{\sum_{i \in \Omega} x_{i}}=s\right\}, & \text { if } e=1 \\ 0, & \text { otherwise }\end{cases}
$$

Note that

$$
\begin{aligned}
\operatorname{deg}\left(\lambda_{\sigma}\right) & =n, \\
\chi\left(\lambda_{\sigma}\right) & =\chi_{n}(\sigma) .
\end{aligned}
$$

Each $\psi \in \Lambda^{*}$ induces a family of maps

$$
\psi_{n}: \mathrm{H}_{n} \rightarrow \mathbb{C}, \quad g \mapsto \psi\left(\lambda_{g}\right) .
$$

In particular, the function $\chi$ defined in (3.1) induces the total sign maps $\chi_{n}$. From now on, we abuse notation and write $\chi$ instead of $\chi_{n}$.

We denote by $\mathrm{H}_{n}^{k}$ the direct product of $k$ copies of $\mathrm{H}_{n}$ and by $\pi_{i}: \mathrm{H}_{n}^{k} \rightarrow \mathrm{H}_{n}$ the projection onto the $i$-th component. For a subgroup $G$ of $\mathrm{H}_{n}^{k}$ we define a $k$-multilinear map $\langle\cdot\rangle_{G}:\left(\Lambda^{*}\right)^{k} \rightarrow \mathbb{C}$ by

$$
\left\langle\psi_{1}, \ldots, \psi_{k}\right\rangle_{G}:=\left\langle\prod_{i=1}^{k} \psi_{i, n}\left(\pi_{i}(\sigma)\right)\right\rangle_{\sigma \in G}=\frac{1}{\# G} \cdot \sum_{\sigma \in G} \prod_{i=1}^{k} \psi_{i}\left(\lambda_{\pi_{i}(\sigma)}\right) .
$$

For example, if $G=\mathrm{H}_{n}^{k}$, we have independence; namely,

$$
\left\langle\psi_{1}, \ldots, \psi_{k}\right\rangle_{G}=\prod_{i=1}^{k}\left\langle\psi_{i}\right\rangle_{\mathrm{H}_{n}} .
$$


Now we describe the connection with arithmetic. Let $K$ be a field of characteristic different from 2 and let $f \in K[T]$ of degree $n$. Suppose that $f$ is separable and $f(0) \neq 0$. If $\omega_{1}, \ldots, \omega_{n}$ is an enumeration of the roots of $f$, then the roots of $f\left(-T^{2}\right)$ are $\pm \rho_{1}, \ldots, \pm \rho_{n}$, where $\rho_{i}$ is a fixed square root of $-\omega_{i}$. The Galois action then induces embeddings $\operatorname{Gal}(f(T) \mid K) \rightarrow S_{n}$ and

$$
\Theta: \operatorname{Gal}\left(f\left(-T^{2}\right) \mid K\right) \rightarrow \mathrm{H}_{n}=V \rtimes S_{n},
$$

where the elements of $S_{n}$ act by permuting the $\omega_{i}$, and the elements of $V$ act by sign change on the $\rho_{i}$, cf. [BBF17, Lemma 3.1]. Over the finite $K=\mathbb{F}_{q}$, the Galois group in (3.6) is generated by the Frobenius automorphism $\phi_{q}: x \mapsto x^{q}$. In fact $\lambda_{f}$ and $\lambda_{\Theta\left(\phi_{q}\right)}$ are equal:

Lemma 3.1. Let $f \in \mathbb{F}_{q}[T]$ monic, square-free and not divisible by $T$. Then $\lambda_{f}=\lambda_{\Theta\left(\phi_{q}\right)}$, where $\phi_{q}$ is the Frobenius automorphism and $\Theta$ is the map from (3.6)

Proof. Let $f=P_{1}^{e_{1}} \cdots P_{r}^{e_{r}}$ be the prime factorization of $f$. Note that by assumption, $\chi_{q}\left(P_{i}\right) \in\{ \pm 1\}$ and $e_{i}=1$ for all $i$, hence $\lambda_{f}(d, e, s)=0=\lambda_{\Theta(\phi)}(d, e, s)$ for all $(d, e, s)$ with either $e>1$ or $s=0$. The set of roots $\Omega=\left\{\omega_{1}, \ldots, \omega_{n}\right\}$ of $f$ is partitioned as $\Omega=\coprod_{i=1}^{r} \Omega_{i}$, where $\Omega_{i}$ is the set of roots of $P_{i}$. Write $\Theta\left(\phi_{q}\right)=x \tau$, and note that the $\Omega_{i}$ are exactly the orbits of $\tau$, of length $\# \Omega_{i}=\operatorname{deg}\left(P_{i}\right)$. By [BBF17, Lemma 3.3], $\chi_{q}\left(P_{i}\right)=(-1)^{\sum_{j \in \Omega_{i}} x_{j}}$. It follows that for any $(d, s)$ with $s \neq 0$,

$$
\begin{aligned}
\lambda_{f}(d, 1, s) & =\#\left\{i: \operatorname{deg}\left(P_{i}\right)=d, e_{i}=1, \chi_{q}\left(P_{i}\right)=s\right\} \\
& =\#\left\{i: \# \Omega_{i}=d,(-1)^{\sum_{j \in \Omega_{i}} x_{j}}=s\right\}=\lambda_{\Theta(\phi)}(d, 1, s) .
\end{aligned}
$$

Thus, $\lambda_{f}(d, e, s)=\lambda_{\Theta(\phi)}(d, e, s)$ for all $(d, e, s)$.

Given $k$ polynomials $f_{1}, \ldots, f_{k} \in K[T]$ that are of degree $n$, separable, with $f_{i}(0) \neq$ 0 , and pairwise coprime, the Galois group $\operatorname{Gal}\left(\prod_{i=1}^{k} f_{i}\left(-T^{2}\right) \mid K\right)$ embeds into $\mathrm{H}_{n}^{k}$. The following proposition computes the limit of correlations of arithmetic functions depending on signed factorization type, where the correlation can be taken over any set of polynomials in $\mathbb{F}_{q}[T]$ that are specializations of a fixed polynomial $f_{A} \in \mathbb{F}_{q}\left[A_{1}, \ldots, A_{m}\right][T]$, where by a specialization of $f_{A}$ we mean the polynomial $f_{a} \in \mathbb{F}_{q}[T]$ obtained by substituting $a_{1}, \ldots, a_{m} \in \mathbb{F}_{q}$ for the variables $A_{1}, \ldots, A_{m}$. For example, the short interval $\left|f-f_{0}\right|<q^{m}$ is the set of specializations of $f_{A}=f_{0}+\sum_{i=0}^{m-1} A_{i} T^{i}$.

Proposition 3.2. Fix $n \geq 1, m \geq 0, k \geq 1$ and $\psi_{1}, \ldots, \psi_{k} \in \Lambda^{*}$. Let $q$ be an odd prime power, let $f_{1, A}, \ldots, f_{k, A} \in \mathbb{F}_{q}\left[A_{1}, \ldots, A_{m}\right][T]$ be monic, of degree $n$, square-free, not divisible by $T$, and pairwise coprime, and denote

$$
g_{A}(T)=\prod_{i=1}^{k} f_{i, A}\left(-T^{2}\right)
$$

and $G=\operatorname{Gal}\left(g_{A} \mid \mathbb{F}_{q}(A)\right) \leq \mathrm{H}_{n}^{k}$. Assume that $G=\operatorname{Gal}\left(g_{A} \mid \overline{\mathbb{F}}_{q}(A)\right)$. Then

$$
\left\langle\prod_{i=1}^{k} \psi_{i, q}\left(f_{i, a}\right)\right\rangle_{a \in \mathbb{F}_{q}^{m}}=\left\langle\psi_{1}, \ldots, \psi_{k}\right\rangle_{G}+O\left(q^{-1 / 2}\right),
$$


where the implied constant depends only on $n, m, k$ and $\psi_{1}, \ldots, \psi_{k}$.

Proof. For $\underline{\lambda}=\left(\lambda_{1}, \ldots, \lambda_{k}\right) \in \Lambda^{k}$ let

$$
A(\underline{\lambda})=\left\{a \in \mathbb{F}_{q}^{m}:\left(\lambda_{f_{1, a}}, \ldots, \lambda_{f_{k, a}}\right)=\underline{\lambda}\right\}
$$

and

and note that

$$
G(\underline{\lambda})=\left\{\sigma \in G:\left(\lambda_{\pi_{1}(\sigma)}, \ldots, \lambda_{\pi_{k}(\sigma)}\right)=\underline{\lambda}\right\}
$$

$$
\prod_{i=1}^{k} \psi_{i, q}\left(f_{i, a}\right)=\prod_{i=1}^{k} \psi_{i}\left(\lambda_{i}\right)=\prod_{i=1}^{k} \psi_{i, n}\left(\pi_{i}(\sigma)\right)
$$

for all $a \in A(\underline{\lambda})$ and $\sigma \in G(\underline{\lambda})$. Thus, since each $\psi \in \Lambda^{*}$ takes only a finite number of values on the set of $\lambda \in \Lambda$ with $\operatorname{deg}(\lambda)=n$, it suffices to prove for each $\underline{\lambda}$ that

$$
\frac{\# A(\underline{\lambda})}{q^{m}}=\frac{\# G(\underline{\lambda})}{\# G}+O\left(q^{-1 / 2}\right) .
$$

Note that the assumption on the $f_{i, A}$ 's can be rephrased as saying that the discriminant $\Delta \in$ $\mathbb{F}_{q}[A]$ of $g_{A}$ is non-zero. Also note that $\Delta(a)$ is the discriminant of $g_{a}(T)=\prod_{i=1}^{k} f_{i, a}\left(-T^{2}\right)$. We distinguish two cases:

If $\lambda_{i}(d, e, s)>0$ for some $i$ and either $e>1$ or $s=0$, then $\lambda_{\pi_{i}(\sigma)}$ equals $\lambda_{i}$ for no $\sigma$, so $\# G(\underline{\lambda})=0$. On the other side, $\lambda_{f_{i, a}}=\lambda_{i}$ only if $\Delta(a)=0$, so as $\operatorname{deg}(\Delta)$ is bounded only in terms of $n$ and $k$, we get $\# A(\underline{\lambda})=O\left(q^{m-1}\right)$, see e.g. [Sch76, Ch. 4 Lemma 3A].

If $\lambda_{i}(d, e, s)=0$ for all $i$ and all $(d, e, s)$ with $e>1$ or $s=0$, then we have embeddings $\Theta_{i, a}: \operatorname{Gal}\left(f_{i, a} \mid \mathbb{F}_{q}\right) \rightarrow \mathrm{H}_{n}$ and $\Theta_{a}: \operatorname{Gal}\left(\prod_{i} f_{i, a} \mid \mathbb{F}_{q}\right) \rightarrow \mathrm{H}_{n}^{k}$, and both Galois groups are generated by the Frobenius automorphism $\phi_{q}$. Applying the Chebotarev theorem [BBF17, Theorem 5.1] to the polynomial $g_{A}$ and the $G$-invariant set $G(\underline{\lambda})$ gives that

$$
\#\left\{a \in \mathbb{F}_{q}^{m}: \Delta(a) \neq 0 \text { and } \Theta_{a}\left(\phi_{q}\right) \in G(\underline{\lambda})\right\}=\frac{\# G(\underline{\lambda})}{\# G} \cdot q^{m}+O\left(q^{m-1 / 2}\right) .
$$

Since $\Theta_{i, a}\left(\phi_{q}\right)=\pi_{i}\left(\Theta_{a}\left(\phi_{q}\right)\right)$, and $\lambda_{f_{i, a}}=\lambda_{\Theta_{i, a}\left(\phi_{q}\right)}$ by Lemma 3.1, we get that the left hand side of (3.7) equals precisely $A(\underline{\lambda})$, which gives the claim.

Next we provide some group theoretical tools to determine $G$ in the case $f_{i, A}=f_{0}+h_{i}+$ $\sum_{j=0}^{m} A_{j} T^{j}$, needed to apply Proposition 3.2 for correlations in short intervals.

3.3. Fiber products of hyperoctahedral groups. For a finite group $G$ we denote by $M(G)$ the intersection over the maximal normal subgroups of $G$. In the literature, $M(G)$ is sometimes called the Melnikov subgroup of $G$. It is the smallest normal subgroup of $G$ with quotient a direct product of simple groups. We will use several times that subgroups of finite elementary abelian $p$-groups are again elementary abelian $p$-groups and therefore products of simple groups. We will also use that if $N \unlhd G$, then $M(N) \subseteq M(G)$, and if $f: G \rightarrow H$ is a homomorphism, then $f(M(G))=M(f(G))$, cf. [F J08, Lemma 25.5.4].

Lemma 3.3. Let $\left(G_{i}\right)_{i \in I}$ be a family of finite groups and let $G:=\prod_{i \in I} G_{i}$. Then $M(G)=$ $\prod_{i \in I} M\left(G_{i}\right)$. 
Proof. For each $i, G_{i} \unlhd G$ implies $M\left(G_{i}\right) \subseteq M(G)$, and therefore $\prod_{i \in I} M\left(G_{i}\right) \subseteq M(G)$. The other inclusion follows as $G / \prod_{i \in I} M\left(G_{i}\right)=\prod_{i \in I} G_{i} / M\left(G_{i}\right)$ is a product of simple groups, so $M(G)=\prod_{i \in I} M\left(G_{i}\right)$.

Proposition 3.4. Let $\left(G_{i}\right)_{i \in I}$ be a finite family of finite groups, put $G=\prod_{i \in I} G_{i}$, let $H \leq G$, and let $\pi_{i}: G \rightarrow G_{i}$ be the projection map. If $H M(G)=G$ and $\pi_{i}(H)=G_{i}$ for all $i$, then $H=G$.

Proof. Suppose first that $I=\{1,2\}$. Then by Goursat's lemma, there exist a group $Q$ and epimorphisms $f_{i}: G_{i} \rightarrow Q$ such that $H$ is the fiber product $G_{1} \times{ }_{Q} G_{2}$. Let $M$ be a maximal normal subgroup of $Q$, put $S=Q / M$, and let $\pi: Q \rightarrow S$ be the quotient map. Then $H$ is contained in the fiber product $G_{1} \times{ }_{S} G_{2}$. Since $M_{i}:=\operatorname{ker}\left(\pi \circ f_{i}\right)$ is a maximal normal subgroup of $G_{i}$, we have

$$
M(G)=M\left(G_{1}\right) \times M\left(G_{2}\right) \subseteq M_{1} \times M_{2} \subseteq G_{1} \times_{S} G_{2} .
$$

Thus,

$$
H M(G) \subseteq G_{1} \times{ }_{S} G_{2} \varsubsetneqq G_{1} \times G_{2},
$$

contradicting the assumption $H M(G)=G$.

The general case now follows by induction: Indeed, for $J \subseteq I$, put $G_{J}=\prod_{i \in J} G_{j}$, let $\pi_{J}: G \rightarrow G_{J}$ be the projection map, and let $H_{J}:=\pi_{J}(H)$. Then $\pi_{i}\left(H_{J}\right)=\pi_{i}(H)=G_{i}$ for all $i \in J$, and

$$
H_{J} M\left(G_{J}\right)=\pi_{J}(H) M\left(\pi_{J}(G)\right) \supseteq \pi_{J}(H M(G))=\pi_{J}(G)=G_{J},
$$

so if $J=I \backslash\left\{i_{0}\right\}$, then the induction hypothesis can be applied to $G_{J}=\prod_{i \in J} G_{i}$ and $H_{J}$, showing that $H_{J}=G_{J}$, and then the case $I=\{1,2\}$ can be applied to $G=G_{J} \times G_{i_{0}}$ and $H$, showing that $H=G$.

Next we compute the Melnikov subgroups of the hyperoctahedral group and of one of its subgroups.

Lemma 3.5. Under the notation of $\S 3.2$, if $n \geq 3$, then

$$
M\left(V \rtimes S_{n}\right)=M\left(V_{n-1} \rtimes S_{n}\right)=V_{n-1} \rtimes A_{n} .
$$

Proof. Let $W$ be either $V_{n}$ or $V_{n-1}$. Write

$$
H=W \rtimes S_{n} \quad \text { and } \quad K=V_{n-1} \rtimes A_{n} \leq H .
$$

It is immediate that $M(H) \subseteq K$, as $K$ is either a maximal normal subgroup of $H$, if $W=V_{n-1}$ or an intersection of such, if $W=V$. To show the converse inclusion, we let $N$ be a maximal normal subgroup of $H$ and aim to show that $K \subseteq N$. Let $A:=H / N$ and $U:=N \cap W$. Then $A$ is a finite simple group and $U$ is an $S_{n}$-invariant subspace of $V$ that is contained in $W$.

If $N$ does not contain $W$, then $W N=H$ from maximality. Thus, $W / U \cong H / N=A$. As $W$ is a 2-elementary abelian group, this implies that $A \cong \mathbb{Z} / 2 \mathbb{Z}$ and that $U$ has index 2 in $W$. By (3.3), since $n \geq 3$ this can happen only if $W=V$ and $U=V_{n-1}$. Hence, from maximality $N=V_{n-1} \rtimes S_{n}$ and thus $K \subseteq N$. 
If $N$ contains $W$, then $N / W$ is a maximal normal subgroup of $H / W \cong S_{n}$. Since $S_{n}$ has a unique maximal normal subgroup, namely $A_{n}$, we conclude that $N=W \rtimes A_{n}$ and so $N$ contains $K$.

For a finite set $I$ we let $\mathrm{H}_{n}^{(I)}$ be the fiber product of copies of $\mathrm{H}_{n}$ with respect to $\chi$; i.e.,

$$
\mathrm{H}_{n}^{(I)}:=\left\{\left(g_{i}\right)_{i \in I} \in \prod_{i \in I} \mathrm{H}_{n}: \chi\left(g_{i}\right)=\chi\left(g_{j}\right) \text { for all } i, j \in I\right\},
$$

and we denote by $\pi_{i}: \mathrm{H}_{n}^{(I)} \rightarrow \mathrm{H}_{n}$ the $i$-projection map. Note that

$$
\prod_{i \in I} V_{n} \rtimes S_{n} \geq \mathrm{H}_{n}^{(I)} \geq \prod_{i \in I} V_{n-1} \rtimes S_{n} \geq \prod_{i \in I} V_{n-1} \rtimes A_{n} .
$$

Lemma 3.6. For $n \geq 3$ and any finite set $I$,

$$
M\left(\prod_{i \in I} V_{n} \rtimes S_{n}\right)=M\left(\mathrm{H}_{n}^{(I)}\right)=M\left(\prod_{i \in I} V_{n-1} \rtimes S_{n}\right)=\prod_{i \in I} V_{n-1} \rtimes A_{n} .
$$

Proof. Let

$$
\Gamma=\prod_{i \in I} V_{n} \rtimes S_{n}, \quad N=\prod_{i \in I} V_{n-1} \rtimes S_{n}, \quad \text { and } \quad K=\prod_{i \in I} V_{n-1} \rtimes A_{n} .
$$

As $N$ is normal in $G$ with abelian quotient and $\chi(x)=1$ for each $x \in V_{n-1} \rtimes S_{n}$, we have that $N \unlhd \mathrm{H}_{n}^{(I)} \unlhd \Gamma$. Hence $M(N) \leq M\left(\mathrm{H}_{n}^{(I)}\right) \leq M(\Gamma)$. Lemmas 3.3 and 3.5 give that $M(\Gamma)=M(N)=K$, which thus proves the claim.

We prove an analogue of Proposition 3.4 for fiber products of $\mathrm{H}_{n}$.

Proposition 3.7. Fix a finite set $I$, put $G=\mathrm{H}_{n}^{(I)}$, and let $H \leq G$. If $H M(G)=G$ and $\pi_{i}(H)=\mathrm{H}_{n}$ for all $i \in I$, then $H=G$.

Proof. Let $N$ and $K$ be as in (3.8). By Lemma 3.6, $M(G)=M(N)=K$. The assumption $H K=H M(G)=G$ implies an isomorphism $G / K \cong H / H \cap K$. In particular, if we put $H_{0}=H \cap N$, then as $N$ is a normal in $G, H_{0}$ is normal in $H$ with $H / H_{0} \cong G / N \cong \mathbb{F}_{2}$ and we have $N=H_{0} K$.

Since $\pi_{i}(H)=\pi_{i}(G)$ and $\left[\pi_{i}(G): \pi_{i}(N)\right]=2$, we conclude that $\pi_{i}\left(H_{0}\right)=\pi_{i}(N)$. Therefore, Proposition 3.4 gives that $H_{0}=N$, from which we conclude that $H=G$.

We shall need the following notation for later use: For a finite family $\mathcal{I}=\left(I_{j}\right)_{j \in J}$ of nonempty finite sets we denote

$$
\mathrm{H}_{n}^{\mathcal{I}}:=\prod_{j \in J} \mathrm{H}_{n}^{\left(I_{j}\right)} \leq \prod_{j \in J} \prod_{i \in I_{j}} \mathrm{H}_{n}
$$


3.4. The Galois group of correlations in short intervals. Let $F$ be a field of characteristic different from 2 , let $n>m \geq 2$ and $r>0$, and let $K=F\left(A_{0}, \ldots, A_{m}\right)$. Let $f_{0} \in F[T]$ be monic of degree $n$ and $h_{1}, \ldots, h_{k} \in F[T]$ of degree less than $n$ and pairwise distinct. Starting from

$$
f(T)=f_{0}(T)+\sum_{i=0}^{m} A_{i} T^{i} \in K[T],
$$

we define $f_{i}(T)=f(T)+h_{i}(T)$ and $f_{h}(T)=\prod_{i=1}^{k} f_{i}(T)$. Note that

$$
f_{i}(0)=f_{0}(0)+A_{0}+h_{i}(0) .
$$

The goal of this section is to determine the Galois group of $f_{h}\left(-T^{2}\right)$.

If $\omega_{1}, \ldots, \omega_{n}$ is an enumeration of the roots of $f_{i}$, then the roots of $f_{i}\left(-T^{2}\right)$ are $\pm \rho_{1}, \ldots, \pm \rho_{n}$, where $\rho_{i}$ is a fixed square root of $-\omega_{i}$. The Galois action induces embeddings $\operatorname{Gal}\left(f_{i}(T) \mid K\right) \hookrightarrow$ $S_{n}$ and $\Theta_{i}: \operatorname{Gal}\left(f_{i}\left(-T^{2}\right) \mid K\right) \hookrightarrow \mathrm{H}_{n}=V \rtimes S_{n}$ as in (3.8).

Proposition 3.8. For each $i$, the action on the roots of $f_{i}(T)$ and $\Theta_{i}$ induce isomorphisms

$$
\operatorname{Gal}\left(f_{i}(T) \mid K\right) \cong S_{n} \quad \text { and } \quad \operatorname{Gal}\left(f_{i}\left(-T^{2}\right) \mid K\right) \cong \mathrm{H}_{n}=V \rtimes S_{n} .
$$

Here, the extensions of $K$ corresponding to the subgroups $V \rtimes A_{n}$ and $V_{n-1} \rtimes S_{n}$ of $\mathrm{H}_{n}$ are $K\left(\sqrt{\operatorname{discr}\left(f_{i}\right)}\right)$ and $K\left(\sqrt{f_{i}(0)}\right)$, respectively.

Proof. See [BBF17, Proposition 4.6] for the claimed isomorphisms. The subgroup $V \rtimes$ $A_{n}$ of $V \rtimes S_{n}$ corresponds to the same extension as the subgroup $A_{n}$ of $S_{n}$, namely to $K\left(\sqrt{\operatorname{discr}\left(f_{i}\right)}\right)$, cf. [Mil14, Corollary 4.2]. Since $f_{i}(0)=(-1)^{n} \omega_{1} \cdots \omega_{n}=\left(\rho_{1} \cdots \rho_{n}\right)^{2}$, the splitting field of $f_{i}\left(-T^{2}\right)$ contains $K\left(\sqrt{f_{i}(0)}\right)$. Since the elements of $V_{n-1}$ act on the roots of $f_{i}\left(-T^{2}\right)$ by an even number of sign changes, $\sqrt{f_{i}(0)}=\rho_{1} \cdots \rho_{n}$ is invariant under $V_{n-1} \rtimes S_{n}$, which implies that $K\left(\sqrt{f_{i}(0)}\right)$ is the field extension of $K$ corresponding to the subgroup $V_{n-1} \rtimes S_{n}$ of $\mathrm{H}_{n}$.

As before we have the maps

$$
\Psi_{i}: \operatorname{Gal}\left(f_{h}\left(-T^{2}\right) \mid K\right) \stackrel{\text { resi }_{i}}{\longrightarrow} \operatorname{Gal}\left(f_{i}\left(-T^{2}\right) \mid K\right) \stackrel{\Theta_{i}}{\rightarrow} \mathrm{H}_{n}, \quad i=1, \ldots, k,
$$

which induce an embedding

$$
\Psi: \operatorname{Gal}\left(f_{h}\left(-T^{2}\right) \mid K\right) \rightarrow \mathrm{H}_{n}^{k} .
$$

Denote by $H$ the image of $\Psi, G=\prod_{i=1}^{k} \mathrm{H}_{n}$, and $\pi_{i}: G \rightarrow \mathrm{H}_{n}$ the projection onto the $i$-th factor. Proposition 3.8 implies that

$$
\pi_{i}(H)=\pi_{i}(G)=\mathrm{H}_{n}, \quad \text { for all } i=1, \ldots, k .
$$

Let

$$
\begin{aligned}
J & =\left\{h_{i}(0): i=1, \ldots, k\right\}, & \\
I_{a} & =\left\{i: h_{i}(0)=a\right\}, & \text { for } a \in J, \\
\mathcal{I}_{h} & =\left(I_{a}\right)_{a \in J} &
\end{aligned}
$$


Recall that a family $\left(x_{1}, \ldots, x_{s}\right)$ of elements of $K^{\times}$is square-independent if the residues in $K^{\times} / K^{\times 2}$ are $\mathbb{F}_{2}$-independent. By Kummer theory, this is equivalent to

$$
\operatorname{Gal}\left(K\left(\sqrt{x_{1}}, \ldots, \sqrt{x_{s}}\right) \mid K\right) \cong\left(\mathbb{F}_{2}\right)^{s} .
$$

Lemma 3.9. The family

$$
\left(f_{0}(0)+A_{0}+a\right)_{a \in J} \cup\left(\operatorname{discr}\left(f_{i}\right)\right)_{i=1, \ldots, k}
$$

is square-independent in $K$.

Proof. By [BB15, Proposition 2.1], the $\operatorname{discr}\left(f_{i}\right)$ are non-squares and pairwise coprime in the ring $R=F\left(A_{1}, \ldots, A_{m}\right)\left[A_{0}\right]$. For $a \in J$ and $i=1, \ldots, k$, writing $f_{i}$ as $\tilde{f}_{i}+A$ with $A=f_{0}(0)+A_{0}+a$ and $\tilde{f}_{i} \in F\left(A_{1}, \ldots, A_{m}\right)[T]$, then $\tilde{f}_{i}$ is separable and [BBF17, Lemma 4.5] gives that $A$ does not divide $\operatorname{discr}\left(f_{i}\right)$ in $R$. Since $A$ is a prime element in the UFD $R$, for each $a \in J$, this shows that the family $\left(f_{0}(0)+A_{0}+a\right)_{a \in J} \cup\left(\operatorname{discr}\left(f_{i}\right)\right)_{i=1, \ldots, r}$ consists of pairwise coprime non-squares in $R$, which implies that it is square-independent in the fraction field $K$ of $R$.

For each $i$, let $K_{i}=K\left(\sqrt{f_{i}(0)}\right)=K\left(\sqrt{f_{0}(0)+A_{0}+h_{i}(0)}\right)$ and let

$$
\operatorname{res}_{K_{i}}: \operatorname{Gal}\left(f_{i}\left(-T^{2}\right) \mid K\right) \rightarrow \operatorname{Gal}\left(K_{i} \mid K\right)
$$

denote the corresponding restriction map.

Proposition 3.10. The map $\Psi$ given in (3.9) induces an isomorphism

$$
\operatorname{Gal}\left(f_{h}\left(-T^{2}\right) \mid K\right) \cong \mathrm{H}_{n}^{\mathcal{I}_{h}},
$$

where $\mathcal{I}_{h}$ is defined in (3.13).

Proof. Let $H$ be the image of $\operatorname{Gal}\left(f_{h}\left(-T^{2}\right) \mid K\right)$ in $G=\prod_{i=1}^{k} \mathrm{H}_{n}=\prod_{a \in J} \prod_{i \in I_{a}} \mathrm{H}_{n}$. As $\Psi$ is injective, it suffices to show that $H=\mathrm{H}_{n}^{\mathcal{I}_{h}}$.

We first treat the case $J=\{a\}$. Since $K_{i}$ is the same for all $i \in I_{a}$, we call it $K_{a}$. It is a common subfield of the splitting fields of all $f_{i}\left(-T^{2}\right)$, hence $H$ is contained in $\mathrm{H}_{n}^{\left(I_{a}\right)}$ We apply Proposition 3.7, where $\pi_{i}(H)=\mathrm{H}_{n}$ by (3.10). By Lemma 3.6,

$$
M\left(\mathrm{H}_{n}^{\left(I_{a}\right)}\right)=\prod_{i \in I_{a}} V_{n-1} \rtimes A_{n},
$$

and $M\left(\mathrm{H}_{n}^{\left(I_{a}\right)}\right) \cap H$ corresponds to the field extension

$$
K_{a} \cdot K\left(\sqrt{\operatorname{discr}\left(f_{i}\right)}, i \in I_{a}\right) .
$$

By Lemma 3.9, $f_{0}(0)+A_{0}+a$ and $\left(\operatorname{discr}\left(f_{i}\right)\right)_{i \in I_{a}}$ are square-independent, which means that $H M\left(\mathrm{H}_{n}^{\left(I_{a}\right)}\right)=\mathrm{H}_{n}^{\left(I_{a}\right)}$. Therefore, we verified the assumptions of Proposition 3.7 and so we conclude that $H=\mathrm{H}_{n}^{\left(I_{a}\right)}$, as needed.

For general $J$, if $\pi_{a^{\prime}}: \prod_{a \in J} \prod_{i \in I_{a}} \mathrm{H}_{a} \rightarrow \prod_{i \in I_{a^{\prime}}} \mathrm{H}_{n}$ denotes the projection map, then $\pi_{a}(H)=\mathrm{H}_{n}^{\left(I_{a}\right)}$ for all $a \in J$ by the previous case, hence $H \leq \prod_{a \in J} \mathrm{H}_{n}^{\left(I_{a}\right)}=\mathrm{H}_{n}^{\mathcal{I}_{h}}$. By 
Lemma 3.3,

$$
M\left(\mathrm{H}_{n}^{\mathcal{I}_{h}}\right)=\prod_{a \in J} M\left(\mathrm{H}_{n}^{\left(I_{a}\right)}\right)=\prod_{a \in J} \prod_{i \in I_{a}} V_{n-1} \rtimes A_{n}
$$

and $M\left(\mathrm{H}_{n}^{\mathcal{I}_{h}}\right) \cap H$ corresponds to the field extension

$$
\prod_{a \in J} K_{a} \cdot K\left(\sqrt{\operatorname{discr}\left(f_{i}\right)}, i=1, \ldots, k\right) .
$$

By Lemma 3.9, the family

$$
\left(f_{0}(0)+A_{0}+a\right)_{a \in J} \cup\left(\operatorname{discr}\left(f_{i}\right)\right)_{i=1, \ldots, k}
$$

is square-independent, which means that $H M\left(\mathrm{H}_{n}^{\mathcal{I}_{h}}\right)=\mathrm{H}_{n}^{\mathcal{I}_{h}}$. Therefore, Proposition 3.4, applied to the groups $\left(\mathrm{H}_{n}^{\left(I_{a}\right)}\right)_{a \in J}$ and the subgroup $H \leq \mathrm{H}_{n}^{\mathcal{I}_{h}}=\prod_{a \in J} \mathrm{H}_{n}^{\left(I_{a}\right)}$, gives that $H=\mathrm{H}_{n}^{\mathcal{I}_{h}}$.

3.5. Correlations of arithmetic functions depending on signed factorization type. We are now ready to prove our general result on correlations in short intervals:

Theorem 3.11. Fix $k \geq 1, \psi_{1}, \ldots, \psi_{k} \in \Lambda^{*}, 1 \geq \epsilon>0$ and $n>2 \epsilon^{-1}$. Then for $q$ an odd prime power, $f_{0} \in \mathbb{F}_{q}[T]$ monic of degree $n$ and $h_{1}, \ldots, h_{k} \in \mathbb{F}_{q}[T]$ of degree less than $n$ and pairwise distinct,

$$
\left\langle\prod_{i=1}^{k} \psi_{i, q}\left(f+h_{i}\right)\right\rangle_{\left|f-f_{0}\right|<\left|f_{0}\right|^{\epsilon}}=\left\langle\psi_{1}, \ldots, \psi_{k}\right\rangle_{\mathrm{H}_{n}^{\mathcal{I}_{h}}}+O_{n, k, \psi}\left(q^{-1 / 2}\right)
$$

where the implied constant depends only on $n, k$ and $\psi_{1}, \ldots, \psi_{k}$, and $\mathcal{I}_{h}$ is defined as in (3.13).

Proof. The short interval $\left|f-f_{0}\right|<\left|f_{0}\right|^{\epsilon}$ is precisely the set of specializations of $f_{A}=f_{0}+$ $\sum_{i=0}^{m-1} A_{i} T^{i}$, with $m=\lceil\epsilon n\rceil$. Setting $f_{A, i}=f_{A}+h_{i}$ and $f_{A, h}=\prod_{i=1}^{k} f_{A, i}$, Proposition 3.10 gives that

$$
\operatorname{Gal}\left(f_{A, h}\left(-T^{2}\right) \mid \mathbb{F}_{q}(A)\right)=\operatorname{Gal}\left(f_{A, h}\left(-T^{2}\right) \mid \overline{\mathbb{F}}_{q}(A)\right)=\mathrm{H}_{n}^{\mathcal{I}_{h}} .
$$

In particular, the $f_{A, i}$ are monic, square-free, not divisible by $T$ and pairwise coprime. Therefore, the claim follows from Proposition 3.2.

We note that in the case where the $\psi_{i}$ depend only on cycle type, i.e. $\psi_{i}(d, 1,1)=$ $\psi_{i}(d, 1,-1)$ for all $d$,

$$
\left\langle\psi_{1}, \ldots, \psi_{k}\right\rangle_{\mathrm{H}_{n}^{\mathcal{I}_{h}}}=\prod_{i=1}^{k}\left\langle\psi_{i}\right\rangle_{\mathrm{H}_{n}}
$$

as we will explain in more detail in Lemma 4.8, and hence

$$
\left\langle\prod_{i=1}^{k} \psi_{i, q}\left(f+h_{i}\right)\right\rangle_{f \in M_{n, q}}=\prod_{i=1}^{k}\left\langle\psi_{i, q}(f)\right\rangle_{f \in M_{n, q}}+O\left(q^{-1 / 2}\right),
$$

which was proven already in [ABR15] (although not stated in such generality). 


\section{Correlations in the LARGe Finite Field Limit}

We now prove Theorem 1.2 in short intervals and compute the correlations of some further examples. Due to Theorem 3.11 all that is left to do is to compute the corresponding averages $\left\langle\psi_{1}, \ldots, \psi_{k}\right\rangle_{\mathrm{H}_{n}^{\mathcal{I}_{h}}}$, which is a purely combinatorial task.

We will use on several occasions the (trivial) principles that if $G_{1}, G_{2}$ are finite groups and $\psi_{i}: G_{i} \rightarrow \mathbb{C}$, then

$$
\left\langle\psi_{1}\left(\sigma_{1}\right) \psi_{2}\left(\sigma_{2}\right)\right\rangle_{\left(\sigma_{1}, \sigma_{2}\right) \in G_{1} \times G_{2}}=\left\langle\psi_{1}(\sigma)\right\rangle_{\sigma \in G_{1}} \cdot\left\langle\psi_{2}(\sigma)\right\rangle_{\sigma \in G_{2}}
$$

and if $\pi: G_{2} \rightarrow G_{1}$ is an epimorphism, then

$$
\left\langle\psi_{1}(\pi(\sigma))\right\rangle_{\sigma \in G_{2}}=\left\langle\psi_{1}(\sigma)\right\rangle_{\sigma \in G_{1}} .
$$

4.1. Autocorrelation of $b$ and $r$. We will start with the autocorrelation of $b$, and then look at the closely related arithmetic function $r$, which counts the number of representations as a sum of two squares. The following general consideration will simplify our arguments:

Lemma 4.1. Let $\psi_{1}, \ldots, \psi_{k} \in \Lambda^{*}$ and let $\mathcal{I}=\left(I_{j}\right)_{j \in J}$ be a partition of $\{1, \ldots, k\}$, i.e. $I_{j} \neq \emptyset$ for all $j$ and $\{1, \ldots, k\}=\coprod_{j \in J} I_{j}$. If $\psi_{1}, \ldots, \psi_{k}$ are all supported on $\chi^{-1}(1)=\{\lambda \in \Lambda$ : $\chi(\lambda)=1\}$, then

$$
\left\langle\psi_{1}, \ldots, \psi_{k}\right\rangle_{\mathrm{H}_{n}^{\mathcal{I}}}=\mathfrak{S}_{\mathcal{I}} \cdot\left\langle\psi_{1}\right\rangle_{\mathrm{H}_{n}} \cdots\left\langle\psi_{k}\right\rangle_{\mathrm{H}_{n}},
$$

where $\mathfrak{S}_{\mathcal{I}}=2^{k-\# J}$.

Proof. Since by assumption $\psi_{i, n}(\sigma)=0$ for $\sigma \in \mathrm{H}_{n} \backslash V_{n-1} \rtimes S_{n}$ and since $\mathrm{H}_{n}^{\mathcal{I}}$ contains $\prod_{i} V_{n-1} \rtimes S_{n}$, we get by (4.1) that

$$
\left\langle\psi_{1}, \ldots, \psi_{k}\right\rangle_{\mathrm{H}_{n}^{\mathcal{I}}}=\frac{1}{\# \mathrm{H}_{n}^{\mathcal{I}}} \sum_{\sigma=\left(\sigma_{i}\right) \in \prod_{i} V_{n-1} \rtimes S_{n}} \prod_{i} \psi_{i}\left(\sigma_{i}\right)=\frac{\#\left(V_{n-1} \rtimes S_{n}\right)^{k}}{\# \mathrm{H}_{n}^{\mathcal{I}}} \cdot \prod_{i=1}^{k}\left\langle\psi_{i}\right\rangle_{V_{n-1} \rtimes S_{n}} .
$$

This finishes the proof as $\mathfrak{S}_{\mathcal{I}}=\frac{\# \mathrm{H}_{n}^{k}}{\# \mathrm{H}_{n}^{I}}$.

The following result gives the autocorrelations of $b_{q}$ in short intervals. Theorem 1.2 is the special case with parameters $\epsilon=1$ and $f_{0}=T^{n}$ :

Theorem 4.2. Fix $k \geq 1,1 \geq \epsilon>0$ and $n>2 \epsilon^{-1}$. Then for $q$ an odd prime power, $f_{0} \in \mathbb{F}_{q}[T]$ monic of degree $n$ and $h_{1}, \ldots, h_{k} \in \mathbb{F}_{q}[T]$ of degree less than $n$ and pairwise distinct,

$$
\begin{aligned}
\left\langle\prod_{i=1}^{k} b_{q}\left(f+h_{i}\right)\right\rangle_{\left|f-f_{0}\right|<\left|f_{0}\right|^{\epsilon}} & =\mathfrak{S}_{q, h} \cdot\left\langle b_{q}(f)\right\rangle_{f \in M_{n, q}}^{k}+O_{n, k}\left(q^{-1 / 2}\right) \\
& =\mathfrak{S}_{h} \cdot \frac{1}{4^{n k}}\left(\begin{array}{c}
2 n \\
n
\end{array}\right)^{k}+O_{n, k}\left(q^{-1 / 2}\right)
\end{aligned}
$$

where the implied constant depends only on $n$ and $k$, and $\mathfrak{S}_{q, h}$ and $\mathfrak{S}_{h}$ are defined as in (1.13) and (1.14). 
Proof. Set $\psi_{1}=\cdots=\psi_{k}=b$ and $\mathcal{I}=\mathcal{I}_{h}$ and note that Lemma 4.1 applies, leading to $\langle b, \ldots, b\rangle_{\mathrm{H}_{n}^{\mathcal{I}_{h}}}=\mathfrak{S}_{h} \cdot\langle b\rangle_{\mathrm{H}_{n}}^{k}$, since $\mathfrak{S}_{\mathcal{I}_{h}}=\mathfrak{S}_{h}$. Ewens' sampling formula gives that $\langle b\rangle_{\mathrm{H}_{n}}=$ $\frac{1}{4^{n}}\left(\begin{array}{c}2 n \\ n\end{array}\right)$, see [BBF17, Lemma 2.3]. Therefore Theorem 3.11 shows that

$$
\left\langle\prod_{i=1}^{k} b_{q}\left(f+h_{i}\right)\right\rangle_{\left|f-f_{0}\right|<\left|f_{0}\right|^{\epsilon}}=\mathfrak{S}_{h} \cdot \frac{1}{4^{n k}}\left(\begin{array}{c}
2 n \\
n
\end{array}\right)^{k}+O_{n, k}\left(q^{-1 / 2}\right) .
$$

In particular, $\left\langle b_{q}(f)\right\rangle_{f \in M_{n, q}}=\frac{1}{4^{n}}\left(\begin{array}{c}2 n \\ n\end{array}\right)+O\left(q^{-1 / 2}\right)$ (which also follows from [BSW16]). Together with $\mathfrak{S}_{h}=\mathfrak{S}_{q, h}+O\left(q^{-1 / 2}\right)$ (Proposition 2.18), we conclude that

$$
\mathfrak{S}_{h} \cdot \frac{1}{4^{n k}}\left(\begin{array}{c}
2 n \\
n
\end{array}\right)^{k}=\mathfrak{S}_{q, h} \cdot\left\langle b_{q}(f)\right\rangle_{f \in M_{n, q}}^{k}+O_{n, k}\left(q^{-1 / 2}\right)
$$

as needed.

Just like the autocorrelation of $b$ (see the introduction), the autocorrelation of $r$ has been studied by various authors, but the latter one turns out to be much more accessible: Already Estermann [Est32] proves an asymptotic formula for $\langle r(n) r(n+h)\rangle_{n \leq x}$. Apparently unaware of that, Connors and Keating [CK97, eqn. (27)] provide a conjectural formula and numerics for $\lim _{x \rightarrow \infty}\langle r(n) r(n+h)\rangle_{n \leq x}$ and observe that here the data seems to match their prediction even better than in the case of $b$. We define the function field analogue of $r$ as

$$
r_{q}(f)=\#\left\{(A, B): f=A^{2}+T B^{2}, A, B \in \mathbb{F}_{q}[T]\right\} /\{ \pm 1\} .
$$

We note that $r_{q}$ is multiplicative and therefore one obtains, just like for $r$, the formula

$$
r_{q}\left(P_{1}^{e_{1}} \cdots P_{r}^{e_{r}}\right)=\left\{\begin{array}{ll}
\prod_{\chi_{q}\left(P_{i}\right)=1}\left(e_{i}+1\right), & \text { if } e_{i} \text { is even for all } i \text { with } \chi_{q}\left(P_{i}\right)=-1 \\
0, & \text { otherwise }
\end{array},\right.
$$

which implies that $r_{q}$ is induced from $r \in \Lambda^{*}$ defined by

$$
r(\lambda)= \begin{cases}\prod_{d, e}(e+1)^{\lambda(d, e, 1)}, & \text { if } \lambda(d, 2 e+1,-1)=0 \text { for all } d, e . \\ 0, & \text { otherwise }\end{cases}
$$

We remark without proof that, like for integers, also the formula

$$
r_{q}(f)=\sum_{d \mid f, d \in M_{q}} \chi_{q}(d)
$$

holds.

We start by computing the mean value of $r$ on $\mathrm{H}_{n}$.

Lemma 4.3. $\langle r\rangle_{\mathrm{H}_{n}}=1$

Proof. As usual we write an element $\sigma \in \mathrm{H}_{n}$ as $\sigma=x \tau$ with $\tau \in S_{n}$ and $x=\left(x_{1}, \ldots, x_{n}\right) \in$ $V=\mathbb{F}_{2}^{n}$ and we recall the corresponding signed factorization type $\lambda_{\sigma}$ attached to $\sigma$ and given in (3.5). Note that $\lambda_{\sigma}(d, e, s)=0$ if $e>1$. Thus, if $\sum_{d} \lambda_{\sigma}(d, 1,-1)=0$, 
then $\sum_{d, e} \lambda_{\sigma}(d, 2 e+1,-1)=0, \prod_{d, e}(e+1)^{\lambda_{\sigma}(d, e, 1)}=2^{\sum_{d} \lambda_{\sigma}(d, 1,1)}$, and $\sum_{d} \lambda_{\sigma}(d, 1,1)=$ $\sum_{d, s} \lambda_{\sigma}(d, 1, s)=\sum_{d} \lambda_{\tau}(d, 1,1)$. By (4.3) we therefore get

$$
r_{n}(\sigma)= \begin{cases}2^{\sum_{d} \lambda_{\tau}(d, 1,1)}, & \text { if } \sum_{d} \lambda_{\sigma}(d, 1,-1)=0 \text { and } \sigma=x \tau \\ 0, & \text { otherwise }\end{cases}
$$

Let $N_{\tau}$ denote the number of $\sigma=x \tau \in \mathrm{H}_{n}$ with $\sum_{d} \lambda_{\sigma}(d, 1,-1)=0$. Note that $\sigma$ is counted by $N_{\tau}$ if and only if for each orbit $I \subset\{1, \ldots, n\}$ of $\tau$ we have $\sum_{i \in I} x_{i}=0$, and so there are $2^{n-j}$ such $\sigma$, where

$$
j=\sum_{d, s} \lambda_{\tau}(d, 1, s)=\sum_{d} \lambda_{\tau}(d, 1,1)
$$

is the number of orbits of $\tau$. So

$$
N_{\tau}=2^{n-\sum_{d} \lambda_{\tau}(d, 1,1)}
$$

By (4.4) and (4.5), we conclude that

$$
\sum_{\sigma \in \mathrm{H}_{n}} r_{n}(\sigma)=\sum_{\tau \in S_{n}} \sum_{x \in \mathbb{F}_{2}^{n}} r_{n}(x \tau)=\sum_{\tau \in S_{n}} N_{\tau} r_{n}(\tau)=\sum_{\tau \in S_{n}} 2^{n}=n ! 2^{n}=\# \mathrm{H}_{n},
$$

and so $\langle r\rangle_{\mathrm{H}_{n}}=1$.

Now we can compute the autocorrelation of $r$ :

Theorem 4.4. Fix $k \geq 1,1 \geq \epsilon>0$ and $n>2 \epsilon^{-1}$. Then for $q$ an odd prime power, $f_{0} \in \mathbb{F}_{q}[T]$ monic of degree $n$ and $h_{1}, \ldots, h_{k} \in \mathbb{F}_{q}[T]$ of degree less than $n$ and pairwise distinct,

$$
\begin{aligned}
\left\langle\prod_{i=1}^{k} r_{q}\left(f+h_{i}\right)\right\rangle_{\left|f-f_{0}\right|<\left|f_{0}\right|^{\epsilon}} & =\mathfrak{S}_{q, h} \cdot\left\langle r_{q}(f)\right\rangle_{f \in M_{n, q}}^{k}+O_{n, k}\left(q^{-1 / 2}\right) \\
& =\mathfrak{S}_{h}+O_{n, k}\left(q^{-1 / 2}\right)
\end{aligned}
$$

where the implied constant depends only on $n$ and $k$, and $\mathfrak{S}_{q, h}$ and $\mathfrak{S}_{h}$ are defined as in (1.13) and (1.14).

Proof. Apply Theorem 3.11 with $\psi_{1}=\cdots=\psi_{k}=r$. Note that Lemma 4.1 applies again, so the claim follow from Lemma 4.3.

4.2. Cross-correlations of $b$ and $r$ with $1_{\mathbb{P}}$. We now turn to cross-correlations of $b$ and $r$ with the prime indicator function $1_{\mathbb{P}}$, which also have been studied by various authors: Starting from a conjecture of Hardy and Littlewood [HL24, Conjecture J] on the number of representations of an integer as the sum of two squares and a prime, [Hoo57, Theorem 2 ] proves an asymptotic formula for

$$
\frac{1}{x} \sum_{p \leq x} r(p+h)=\left\langle r(n) 1_{\mathbb{P}}(n-h)\right\rangle_{n \leq x}
$$


under the Extended Riemann Hypothesis, which [Bre62] then proves unconditionally. Motohashi in [Mot70, Conjecture $\mathrm{J}^{*}$, Theorem 2] and [Mot71] gives a conjectural asymptotic formula for

$$
\frac{1}{x} \sum_{p \leq x} b(p-1)=\left\langle b(n) 1_{\mathbb{P}}(n+1)\right\rangle_{n \leq x-1}
$$

and proves upper and lower bounds. Iwaniec [Iwa72, p. 204] also proves lower and upper bounds and suggests a correction of Motohashi's conjecture. We now give a function field version of the Motohashi-Iwaniec conjecture (or rather a generalization of it from $h=1$ to arbitrary $h$ ) and prove it in the large finite field limit.

The heuristics for the cross-correlation of $b$ and $1_{\mathbb{P}}$ is very similar to that of the autocorrelation of $b$ discussed in Section 2, hence we omit some of the details, and leave them as an exercise for the reader. Let $h \in \mathbb{F}_{q}[T]$ be non-zero. For each prime polynomial $P$, we let

$$
\rho_{q, h}(P)=\lim _{\nu \rightarrow \infty} \frac{\#\left\{f \bmod P^{\nu}: f \in M_{q} \text { prime and } \exists A, B: f+h \equiv A^{2}+T B^{2} \bmod P^{\nu}\right\}}{|P|^{\nu}},
$$

$$
\rho_{q}(P)=\lim _{\nu \rightarrow \infty} \frac{\#\left\{(f, g) \bmod P^{\nu}: f \in M_{q} \text { prime and } \exists A, B: g \equiv A^{2}+T B^{2} \bmod P^{\nu}\right\}}{|P|^{2 \nu}} .
$$

One may verify that the limits indeed exist. So $\rho_{q, h}(P) / \rho_{q}(P)$ measures the local deviation at $P$ from the random model. One then may make the following

Conjecture 4.5. Fix $N \geq 1$. Then for $q$ an odd prime power, $n \geq N$, and nonzero $h \in \mathbb{F}_{q}[T]$ of degree less than $N$,

$$
\begin{aligned}
\left\langle 1_{\mathbb{P}}(f) b_{q}(f+h)\right\rangle_{f \in M_{n, q}} & \sim \mathfrak{T}_{q, h} \cdot\left\langle 1_{\mathbb{P}}(f)\right\rangle_{f \in M_{n, q}} \cdot\left\langle b_{q}(f)\right\rangle_{f \in M_{n, q}} \\
& \sim \mathfrak{T}_{q, h} \cdot K_{q} \cdot \frac{1}{n 4^{n}}\left(\begin{array}{c}
2 n \\
n
\end{array}\right),
\end{aligned}
$$

uniformly as $q^{n} \rightarrow \infty$, where $K_{q}$ is defined as in (1.12) and

$$
\mathfrak{T}_{q, h}=\prod_{\substack{P \in \mathbb{F}_{q}[T] \\ \text { monic irred. }}} \frac{\rho_{q, h}(P)}{\rho_{q}(P)} .
$$

We note that $\mathfrak{T}_{q, h}$ converges. Next we give formulas for the $\rho$ 's. Note that $f$ is congruent to a prime modulo $P^{\nu}$ if and only if either $f \not \equiv 0 \bmod P$ or $f \equiv P \bmod P^{\nu}$, and therefore replacing the condition " $f$ prime" by " $f \not \equiv 0 \bmod P$ " leads to the same limits and at the same time simplifies the computations.

Lemma 4.6. Let $P \in \mathbb{F}_{q}[T]$ monic irreducible.

(1) $\rho_{q}(P)=\left(1-|P|^{-1}\right) \delta_{q, 0}(P)$ (Recall that $\delta_{q, 0}(P)$ is explicitly given by Corollary 2.6.)

(2) If $\chi(P)=1$, then $\rho_{q, h}(P)=\rho_{q}(P)=1-|P|^{-1}$. 
(3) If $\chi(P)=-1$, then

$$
\rho_{q, h}(P)= \begin{cases}1-|P|^{-1}, & \text { if } P \mid h \\ 1-|P|^{-1}-(1+|P|)^{-1}, & \text { otherwise. }\end{cases}
$$

(4) If $\chi(P)=0$, then

$$
\rho_{q, h}(P)=\frac{1}{2}-\frac{1}{2 q}\left(1+\chi_{q}(h)\right) .
$$

Proof. (1) is immediate and (2) follows directly from Lemma 2.3(3) while (3) and (4) follows from Lemma 2.3 using similar arguments as those used in the proof of Proposition 2.15.

Just like we deduced Proposition 2.18 from Proposition 2.8 and Corollary 2.6, we can use Lemma 4.6 to conclude that

$$
\mathfrak{T}_{q, h}=1+O\left(q^{-1}\right)
$$

where the implied constant depends only on the degree of $h$. Since also $K_{q}=1+O\left(q^{-1}\right)$, the next results proves Conjecture 4.5 in the large finite field limit.

Theorem 4.7. Fix $1 \geq \epsilon>0$ and $n>2 / \epsilon$. Then for $q$ an odd prime power, $f_{0} \in \mathbb{F}_{q}[T]$ monic of degree $n$ and $h \in \mathbb{F}_{q}[T]$ of degree less than $n$,

$$
\begin{aligned}
\left\langle 1_{\mathbb{P}}(f) b_{q}(f+h)\right\rangle_{\left|f-f_{0}\right|<\left|f_{0}\right|^{\epsilon}} & =\mathfrak{T}_{q, h} \cdot\left\langle 1_{\mathbb{P}}(f)\right\rangle_{f \in M_{n, q}} \cdot\left\langle b_{q}(f)\right\rangle_{f \in M_{n, q}}+O_{n}\left(q^{-1 / 2}\right) \\
& =\frac{1}{n 4^{n}}\left(\begin{array}{c}
2 n \\
n
\end{array}\right)+O_{n}\left(q^{-1 / 2}\right)
\end{aligned}
$$

where $\mathfrak{T}_{q, h}$ is defined as in (4.8) and the implied constant depends only on $n$.

Proof. Apply Theorem 3.11 with $k=2, h_{1}=0, h_{2}=-h, \psi_{1}=b, \psi_{2}=1_{\mathbb{P}}$. Note that $\psi_{2}$ satisfies the assumption of the following lemma, which proves the first equality. For the second equality use that $\mathfrak{T}_{q, h}=1+O\left(q^{-1}\right)$ and $\langle b\rangle_{\mathrm{H}_{n}}=\frac{1}{4^{n}}\left(\begin{array}{c}2 n \\ n\end{array}\right)$ (see above), and that $\left\langle 1_{\mathbb{P}}\right\rangle_{\mathrm{H}_{n}}=\frac{1}{n}$, as this is the fraction of $n$-cycles in $S_{n}$.

Lemma 4.8. Let $\psi_{1}, \ldots, \psi_{k} \in \Lambda^{*}$ and let $\mathcal{I}=\left(I_{j}\right)_{j \in J}$ be a partition of $\{1, \ldots, k\}$ as above. If $\psi_{k}$ depends only on cycle type in the sense that $\psi_{k}(d, 1,1)=\psi_{k}(d, 1,-1)$ for all $d$, then

$$
\left\langle\psi_{1}, \ldots, \psi_{k}\right\rangle_{\mathrm{H}_{n}^{\mathcal{I}}}=\left\langle\psi_{1}, \ldots, \psi_{k-1}\right\rangle_{\mathrm{H}_{n}^{\mathcal{I}^{\prime}}} \cdot\left\langle\psi_{k}\right\rangle_{\mathrm{H}_{n}},
$$

where $\mathcal{I}^{\prime}=\left(I_{j}^{\prime}\right)_{j \in J^{\prime}}, I_{j}^{\prime}=I_{j} \backslash\{k\}, J^{\prime}=\left\{j \in J: I_{j}^{\prime} \neq \emptyset\right\}$.

Proof. Let $\pi: \mathrm{H}_{n} \rightarrow S_{n}$ be the quotient map. The assumption implies that $\psi_{k, n}=\tilde{\psi}_{k, n} \circ \pi$ with a function $\tilde{\psi}_{k, n}: S_{n} \rightarrow \mathbb{C}$. Observe that restricting the homomorphism

$$
\mathrm{id}_{\mathrm{H}_{n}^{\mathcal{I}^{\prime}}} \times \pi: \mathrm{H}_{n}^{\mathcal{I}^{\prime}} \times \mathrm{H}_{n} \rightarrow \mathrm{H}_{n}^{\mathcal{I}^{\prime}} \times S_{n}
$$


gives an epimorphism $\mathrm{H}_{n}^{\mathcal{I}} \rightarrow \mathrm{H}_{n}^{\mathcal{I}^{\prime}} \times S_{n}$. Thus,

$$
\begin{aligned}
\left\langle\psi_{1}, \ldots, \psi_{k}\right\rangle_{\mathrm{H}_{n}^{\mathcal{I}}} & \stackrel{(4.2)}{=}\left\langle\prod_{i=1}^{k-1} \psi_{i, n}\left(\pi_{i}(\sigma)\right) \cdot \tilde{\psi}_{k, n}\left(\pi_{k}(\sigma)\right)\right\rangle_{\sigma \in \mathrm{H}_{n}^{\mathcal{I}^{\prime}} \times S_{n}} \\
& \stackrel{(4.1)}{=}\left\langle\prod_{i=1}^{k-1} \psi_{i, n}\left(\pi_{i}(\sigma)\right)\right\rangle_{\sigma \in \mathrm{H}_{n}^{\mathcal{I}^{\prime}}} \cdot\left\langle\tilde{\psi}_{k, n}(\sigma)\right\rangle_{\sigma \in S_{n}} \\
& \stackrel{(4.2)}{=}\left\langle\psi_{1}, \ldots, \psi_{k-1}\right\rangle_{\mathrm{H}_{n}^{\mathcal{I}^{\prime}}} \cdot\left\langle\psi_{k}\right\rangle_{\mathrm{H}_{n}} .
\end{aligned}
$$

Note that although in Theorem 4.7 the functions $b_{q}$ and $1_{\mathbb{P}}$ become independent in the large finite field limit, this could not have been deduced from the earlier results in [ABR15] (see the remark after Theorem 3.11), as only one of the two arithmetic functions, namely $1_{\mathbb{P}}$, depends only on cycle type, while the other one does not.

4.3. Autocorrelations of $d_{r} \chi$. Let $d_{r}(n)$ be the number of ways to write $n$ as a product of $r$ positive integers. In particular, $d_{2}=\tau$ is the usual divisor function. The problem of estimating the autocorrelations of $d_{r}$, sometimes referred to as 'additive divisor problem', 'shifted divisor', or 'shifted convolution', is well studied both in number fields (see e.g. [CG01, KGH07, CK16] and the recent survey [Tao16]) and function fields (see [ABR15]). The asymptotic of the cross-correlations of the divisor functions are related to computing the moments of the zeta function on the critical line, see [Ivi97]. We consider a twisted version of this; namely, we twist $d_{r}$ by a quadratic character $\chi$ and study the cross-correlations of the $d_{r} \chi$. This is closely related to the moments of the corresponding $L$-function $L(s, \chi)$ on the critical line, but we do not elaborate on this any further, since the goal of this section is to provide yet another application of Theorem 3.11.

In the function field setting, we let $d_{r, q}(f)$ denote the number of ways to write $f$ as a product of $r$ monic polynomials, and we twist $d_{r, q}$ by $\chi_{q}$, the quadratic character modulo $T$. We note that in this setting, computing the moments of the corresponding L-function $L_{q}\left(s, \chi_{q}\right)$ is trivial, since $L_{q}(s, \chi)$ is identically equal to 1 , but the cross-correlations of the $d_{r, q} \chi_{q}$ are nevertheless interesting and, to the best of our knowledge, unknown. We now compute these cross-correlations in the large finite field limit, which we can do as $d_{r, q}$ is induced from $d_{r} \in \Lambda^{*}$ given by

$$
d_{r}(\lambda)=\prod_{d, e, s}\left(\begin{array}{c}
e+r-1 \\
r-1
\end{array}\right)^{\lambda(d, e, s)}
$$

Lemma 4.9. For every $r$ and $n$,

$$
\left\langle d_{r}\right\rangle_{\mathrm{H}_{n}}=\left(\begin{array}{c}
n+r-1 \\
r-1
\end{array}\right) .
$$


Proof. Since $d_{r, n}$ factors through $\pi: \mathrm{H}_{n} \rightarrow S_{n}$, we have

$$
\left\langle d_{r}\right\rangle_{\mathrm{H}_{n}}=\frac{1}{2^{n} n !} \sum_{\sigma \in \mathrm{H}_{n}}\left(\begin{array}{c}
1+r-1 \\
r-1
\end{array}\right)^{\sum_{d, s} \lambda_{\sigma}(d, 1, s)}=\frac{1}{n !} \sum_{\tau \in S_{n}} r^{\omega(\tau)}
$$

where $\omega(\tau)$ is the number of cycles of $\tau$. Viewing $r^{\omega(\tau)}$ as the number of partitions of $\{1, \ldots, n\}$ into $r$ sets that are unions of orbits of $\tau$ and changing order of summation we get that

$$
\sum_{\tau \in S_{n}} r^{\omega(\tau)}=\sum_{\{1, \ldots, n\}=\coprod_{i=1}^{r}} \prod_{A_{i}}^{r} \# A_{i} !
$$

Splitting the sum according to the cardinality of $A_{1}$ and applying induction on $r$ we conclude

$$
\begin{aligned}
\sum_{\{1, \ldots, n\}=\coprod_{i=1}^{r}} \prod_{A_{i}}^{r} \# A_{i} ! & =\sum_{a=0}^{n}\left(\begin{array}{l}
n \\
a
\end{array}\right) a !(n-a) !\left(\begin{array}{c}
n-a+r-2 \\
r-2
\end{array}\right) \\
& =n ! \sum_{a=0}^{n}\left(\begin{array}{c}
a+r-2 \\
r-2
\end{array}\right)=n !\left(\begin{array}{c}
n+r-1 \\
r-1
\end{array}\right) .
\end{aligned}
$$

Lemma 4.10. Let $\psi \in \Lambda^{*}$ with $\psi(d, 1,1)=\psi(d, 1,-1)$ for all $d$. Then $\langle\psi \chi\rangle_{\mathrm{H}_{n}}=0$.

Proof. Write $\psi_{n}=\tilde{\psi}_{n} \circ \pi$ with $\pi: \mathrm{H}_{n} \rightarrow S_{n}$ the quotient map. Then

$$
\sum_{\sigma \in \mathrm{H}_{n}} \psi_{n}(\sigma) \chi_{n}(\sigma)=\sum_{\tau \in S_{n}} \sum_{\sigma \in \pi^{-1}(\tau)} \psi_{n}(\sigma) \chi_{n}(\sigma)=\sum_{\tau \in S_{n}} \tilde{\psi}_{n}(\tau) \sum_{\sigma \in \pi^{-1}(\tau)} \chi_{n}(\sigma)=0
$$

since $\chi_{n}(\sigma)=1$ for half of the $2^{n}$ many $\sigma$ in each $\pi^{-1}(\tau)$, and $\chi_{n}(\sigma)=-1$ for the other half.

Lemma 4.11. Let $\psi_{1}, \ldots, \psi_{k} \in \Lambda^{*}$ and let $\mathcal{I}=\left(I_{j}\right)_{j \in J}$ be a partition of $\{1, \ldots, k\}$. If $\psi_{i}(d, 1,1)=\psi_{i}(d, 1,-1)$ for all $i$ and $d$, then

$$
\left\langle\psi_{1} \chi, \ldots, \psi_{k} \chi\right\rangle_{\mathrm{H}_{n}^{I}}=\left\{\begin{array}{ll}
\left\langle\psi_{1}\right\rangle_{\mathrm{H}_{n}} \cdots\left\langle\psi_{k}\right\rangle_{\mathrm{H}_{n}}, & \text { if } \# I_{j} \text { is even for all } j \in J \\
0, & \text { otherwise }
\end{array} .\right.
$$

Proof. First observe that since $\mathrm{H}_{n}^{\mathcal{I}}=\prod_{j \in J} \mathrm{H}_{n}^{\left(I_{j}\right)}$, by principle (4.1) it suffices to prove the claim in the case $\# J=1$, which we therefore assume now. Note that $\left\langle\psi_{i} \chi\right\rangle_{\mathrm{H}_{n}}=0$ by Lemma 4.10 and thus $\left\langle\psi_{i}(\chi+1)\right\rangle_{\mathrm{H}_{n}}=\left\langle\psi_{i}\right\rangle_{\mathrm{H}_{n}}$. So since $\psi_{i}(\chi+1)$ satisfies the assumptions 
of Lemma 4.1 and $\psi_{i}$ satisfies the assumptions of Lemma 4.8, we get

$$
\begin{aligned}
\left\langle\psi_{1} \chi, \ldots, \psi_{k} \chi\right\rangle_{\mathrm{H}_{n}^{\mathcal{I}}} & =\left\langle\psi_{1}(\chi+1)-\psi_{1}, \ldots, \psi_{k}(\chi+1)-\psi_{k}\right\rangle_{\mathrm{H}_{n}^{\mathcal{I}}} \\
& =\prod_{i=1}^{k}\left\langle-\psi_{i}\right\rangle_{\mathrm{H}_{n}}+\sum_{\emptyset \neq S \subseteq\{1, \ldots, k\}}\left(\prod_{i \notin S}\left\langle-\psi_{i}\right\rangle_{\mathrm{H}_{n}} \cdot 2^{\# S-1} \prod_{i \in S}\left\langle\psi_{i}(\chi+1)\right\rangle_{\mathrm{H}_{n}}\right) \\
& =(-1)^{k} \cdot \prod_{i=1}^{k}\left\langle\psi_{i}\right\rangle_{\mathrm{H}_{n}} \cdot\left(1+\sum_{\emptyset \neq S \subseteq\{1, \ldots, k\}}(-1)^{\# S} 2^{\# S-1}\right) .
\end{aligned}
$$

Now note that

$$
\sum_{S \subseteq\{1, \ldots, k\}}(-1)^{\# S} 2^{\# S}=\sum_{S \subseteq\{1, \ldots, k\}} \sum_{S_{0} \subseteq S}(-1)^{\# S}=\sum_{S_{0} \subseteq\{1, \ldots, k\}} \sum_{S_{0} \subseteq S \subseteq\{1, \ldots, k\}}(-1)^{\# S}
$$

and $\sum_{S_{0} \subseteq S \subseteq\{1, \ldots, k\}}(-1)^{\# S}$ equals 0 except if $S_{0}=\{1, \ldots, k\}$, in which case it equals $(-1)^{k}$. Thus,

$$
\left\langle\psi_{1} \chi, \ldots, \psi_{k} \chi\right\rangle_{\mathrm{H}_{n}^{\mathcal{I}}}=(-1)^{k} \cdot \prod_{i=1}^{k}\left\langle\psi_{i}\right\rangle_{\mathrm{H}_{n}} \cdot\left(1+\frac{1}{2}\left((-1)^{k}-1\right)\right),
$$

from which the claim follows.

Theorem 4.12. Fix $k \geq 1, r_{1}, \ldots, r_{k} \geq 2,1 \geq \epsilon>0$ and $n>2 \epsilon^{-1}$. Then for $q$ an odd prime power, $f_{0} \in \mathbb{F}_{q}[T]$ monic of degree $n$ and $h_{1}, \ldots, h_{k} \in \mathbb{F}_{q}[T]$ of degree less than $n$ and pairwise distinct,

$$
\begin{aligned}
\left\langle\prod_{i=1}^{k} d_{r_{i}, q} \chi_{q}\left(f+h_{i}\right)\right\rangle_{\left|f-f_{0}\right|<\left|f_{0}\right|^{\epsilon}} & =\mathfrak{D}_{h} \cdot \prod_{i=1}^{k}\left\langle d_{r_{i}, q}(f)\right\rangle_{f \in M_{n, q}}+O\left(q^{-1 / 2}\right) \\
& =\mathfrak{D}_{h} \cdot \prod_{i=1}^{k}\left(\begin{array}{c}
n+r_{i}-1 \\
r_{i}-1
\end{array}\right)+O\left(q^{-1 / 2}\right)
\end{aligned}
$$

where the implied constant depends only on $n$ and $r_{1}, \ldots, r_{k}$, and

$$
\mathfrak{D}_{h}= \begin{cases}1, & \text { if } \#\left\{i: h_{i}(0)=a\right\} \text { is even for all } a \in \mathbb{F}_{q} \\ 0, & \text { otherwise }\end{cases}
$$

Proof. Apply Theorem 3.11 with $\psi_{i}=d_{r_{i}} \chi$. Note that $d_{r_{1}}, \ldots, d_{r_{k}}$ satisfy the assumptions of Lemma 4.11, and their averages are given by Lemma 4.9 .

\section{ACKNOWLEDGEMENTS}

The authors are very grateful to Ofir Gorodetsky for sharing his signed factorization type viewpoint on sums of two squares with them. They would also like to thank Efrat Bank for helpful discussions on the topic of this work, Hung Bui for suggesting to study correlations relating to moments of $L$-functions, Jon Keating and Zeev Rudnick for their 
advice on a preliminary version of the paper, and Tristan Freiberg, Pär Kurlberg and Lior Rosenzweig for making their preliminary manuscript available to them.

The first author is partially supported by the Israel Science Foundation (grant No. 925/14), the second author by a research grant from the Ministerium für Wissenschaft, Forschung und Kunst Baden-Württemberg.

\section{REFERENCES}

[ABR15] J. C. Andrade, Lior Bary-Soroker, and Zeev Rudnick. Shifted convolution and the Titchmarsh divisor problem over $\mathbb{F}_{q}[t]$. Philosophical Transactions of the Royal Society of London A: Mathematical, Physical and Engineering Sciences, Theo Murphy meeting issue 'Number fields and function fields: coalescences, contrasts and emerging applications' compiled and edited by J. P. Keating, Z. Rudnick and T. D. Wooley, 373(2040), 2015. 1.3, 3.5, 4.2, 4.3

[BB15] E. Bank and L. Bary-Soroker. Prime polynomial values of linear functions in short intervals. $J$. Number Theory 151:263-275, 2015. 1.3, 3.4

[BBF17] E. Bank, L. Bary-Soroker and A. Fehm. Sums of two squares in short intervals in polynomial rings over finite fields. To appear in American J. Math., 2017. 1.2, 1.4, 3.2, 3.2, 3.2, 3.2, 3.4, 3.4, 4.1

[Ban86] G. Bantle. An asymptotic formula for B-twins. Acta Arithmetica 47:297-312, 1986. 1.2

[BSW16] L. Bary-Soroker, Y. Smilansky, and A. Wolf. On the function field analogue of Landau's theorem on sums of squares. Finite Fields Appl. 39:195-215, 2016 1.4, 2.1, 2.1, 4.1

[Bre62] B. M. Bredihin. Binary additive problems with prime numbers. Dokl. Akad. Nauk SSSR 142:766768, 1962. 4.2

[Car15] D. Carmon. The autocorrelation of the Möbius function and Chowla's conjecture for the rational function field in characteristic 2. Philosophical Transactions of the Royal Society of London A: Mathematical, Physical and Engineering Sciences, Theo Murphy meeting issue 'Number fields and function fields: coalescences, contrasts and emerging applications' compiled and edited by J. P. Keating, Z. Rudnick and T. D. Wooley, 373(2040), 2015. 1.3

[CR14] D. Carmon and Z. Rudnick. The autocorrelation of the Möbius function and Chowla's conjecture for the rational function field. Q. J. Math. 65(1):53-61, 2014. 1.3, 4

[CD87] T. Cochrane and R. E. Dressler. Consecutive triples of sums of two squares. Archiv Math. 49:301304, 1987. 1.2

[CK97] R. D. Connors and J. P. Keating. Two-point spectral correlations for the square billiard J. Phys. A: Math. Gen. 30:1817-1830, 1997. 1.2, 2.4.1, 4.1

[CG01] J. B. Conrey and S. M. Gonek. High moments of the Riemann zeta-function. Duke Math. J. 107(3):577-604, 2001. 4.3

[CK16] B. Conrey and J. P. Keating. Moments of zeta and correlations of divisor-sums: IV. Res. Number Theory 2, 2016. 4.3

[Est32] T. Estermann. An asymptotic formula in the theory of numbers. Proc. London Math. Soc. 34:280292, 1932. 4.1

[FJ08] M. D. Fried and M. Jarden. Field Arithmetic. Third Edition. Springer, 2008. 3.3

[FKR17] T. Freiberg, P. Kurlberg and L. Rosenzweig. Poisson distribution for gaps between sums of two squares and level spacings for toral point scatterers. arXiv:1701.01157 [math-ph], 2017. 1.2, 1.2, $2.2,2.3,2.3$

[Gor16] O. Gorodetsky. A Polynomial Analogue of Landau's Theorem and Related Problems. arXiv:1603.02890 [math.NT], 2016. 1.4

[Hal06] C. Hall. L-functions of twisted Legendre curves. J. Number Theory 119(1):128-147, 2006. 1 
[HL24] G. H. Hardy and J. E. Littlewood. Some Problems of 'Partitio Numerorum'(V): A Further Contribution to the Study of Goldbach's Problem. Proc. London Math. Soc. S2-22 no. 1, 46-56, 1924. 4.2

[Hoo57] C. Hooley. On the representation of a number as the sum of two squares and a prime. Acta Math. 97:189-210, 1957. 4.2

[Hoo71] C. Hooley. On the intervals between numbers that are sums of two squares. Acta Math. 127(1):279-297, 1971. 1.2

[Hoo73] C. Hooley. On the intervals between numbers that are sums of two squares. II. J. Number Theory 5:215-217, 1973. 1.2

[Hoo74] C. Hooley. On the intervals between numbers that are sums of two squares. III. J. Reine Angew. Math. 267(1):207-218, 1974. 1.2, 1.2

[Hoo94] C. Hooley. On the intervals between numbers that are sums of two squares. IV. J. Reine Angew. Math. 452:79-109, 1994. 1.2

[IS72] K.-H. Indlekofer and W. Schwarz. Über B-Zwillinge. Archiv Math. 23:251-256, 19721.2

[Ind74] K.-H. Indlekofer. Scharfe untere Abschätzung für die Anzahlfunktion der B-Zwillinge. Acta Arithmetica 26:207-212, 1974. 1.2

[Ivi97] A. Ivic. The general additive divisor problem and moments of the zeta-function. New trends in probability and statistics $4: 69-89,1997.4 .3$

[Iwa72] H. Iwaniec. Primes of the type $\varphi(x, y)+A$ where $\varphi$ is a quadratic form. Acta Arithmetica 21:203234, 1972. 4.2

[Iwa76] H. Iwaniec. The half dimensional sieve. Acta Arithmetica 29:69-95, 1976. 1.2, 1.2

[KGH07] J. P. Keating, S. M. Gonek, and C. P. Hughes. A hybrid Euler-Hadamard product for the Riemann zeta function. Duke Math. J 136:507-549, 2007. 4.3

[KR16] J. P. Keating and E. Roditty-Gershon. Arithmetic correlations over large finite fields. Int. Math. Res. Not. IMRN 2016, no. 3, 860-874, 2016. 1.5

[KR14] J. P. Keating and Z. Rudnick. The variance of the number of prime polynomials in short intervals and in residue classes. Int. Math. Res. Not. IMRN 2014(1):259-288, 2014. 2.1, 2

[Kel78] P. J. Kelly. The number of B-twins in an interval. Dissertation, Nottingham, 1978. 1.2

[Lan08] E. Landau. Über die Einteilung der positiven ganzen Zahlen in vier Klassen nach der Mindestzahl der zu ihrer additiven Zusammensetzung erforderlichen Quadrate. Arch. Math. Phys. 13:305-312, 1908. 1.2

[Mil14] J. Milne. Fields and Galois theory. Lecture notes, version 4.50, 2014. 3.4

[Mot70] Y. Motohashi. On the distribution of prime numbers which are of the form $x^{2}+y^{2}+1$. Acta Arithmetica XVI:351-363, 1970. 4.2

[Mot71] Y. Motohashi. On the distribution of prime numbers which are of the form " $x^{2}+y^{2}+1$ ". II. Acta Math. Acad. Sci. Hungar. 22:207-210, 1971. 4.2

[Pol08] P. Pollack. Simultaneous prime specializations of polynomials over finite fields. Proc. Amer. Math. Soc. 136(11):3775-3784, 2008. 1.3

[Poo03] B. Poonen. Squarefree values of multivariable polynomials. Duke Math. J. 118(2):353-373, 2003. 1

[Rie65] G. J. Rieger. Aufeinanderfolgende Zahlen als Summen von zwei Quadraten. Indag. Math. 27:208220, 1965. 1.2

[Ros02] M. Rosen. Number Theory in Function Fields. Springer, 2002. 2.3

[Rud14] Z. Rudnick. Some problems in analytic number theory for polynomials over a finite field. Proceedings of the ICM vol 1, 2014. 1.3

[Sage] SageMath, the Sage Mathematics Software System (Version SageMath-7.2.beta0), The Sage Developers, 2016, http://www. sagemath.org. 2.4

[Sch76] W. M. Schmidt. Equations over Finite Fields. An Elementary Approach. Springer 1976. 3.2

[Sch72] W. Schwarz. Über B-Zwillinge II. Archiv Math. 23:408-409, 1972. 1.2 
[Smi13] Y. Smilansky. Sums of two squares - pair correlation \& distribution in short intervals. Int. J. Number Theory 09, 2013. 1.2

[Tao16] T. Tao. Heuristic computation of correlations of higher order divisor functions. WordPress.com, What's new, Online Blog. http://goo.gl/GBncWw 4.3

Raymond and Beverly Sackler School of Mathematical Sciences, Tel Aviv University, Tel Aviv 69978, IsRAel

E-mail address: barylior@post.tau.ac.il

School of Mathematics, Alan Turing Building, The University of Manchester, Oxford Road, Manchester, M13 9PL, United Kingdom

E-mail address: arno.fehm@manchester.ac.uk 